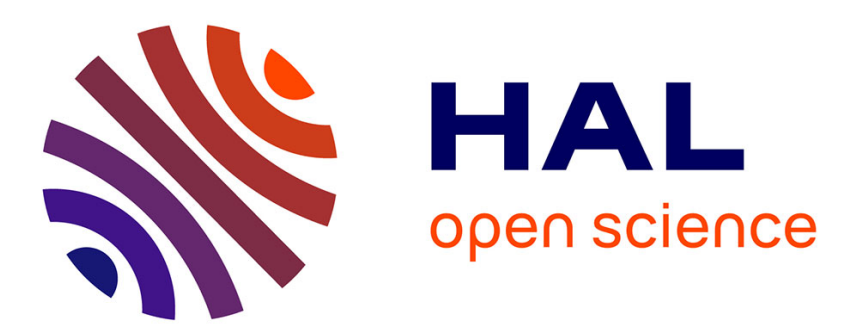

\title{
Multivariate pattern analysis of fMRI data for imaginary and real colours in grapheme-colour synaesthesia
}

\author{
Mathieu J Ruiz, Michel Dojat, Jean-Michel Hupé
}

\section{To cite this version:}

Mathieu J Ruiz, Michel Dojat, Jean-Michel Hupé. Multivariate pattern analysis of fMRI data for imaginary and real colours in grapheme-colour synaesthesia. European Journal of Neuroscience, 2020, 52 (5), pp.3434-3456. 10.1111/ejn.14774 . hal-03020119

\section{HAL Id: hal-03020119 https://hal.science/hal-03020119}

Submitted on 23 Nov 2020

HAL is a multi-disciplinary open access archive for the deposit and dissemination of scientific research documents, whether they are published or not. The documents may come from teaching and research institutions in France or abroad, or from public or private research centers.
L'archive ouverte pluridisciplinaire HAL, est destinée au dépôt et à la diffusion de documents scientifiques de niveau recherche, publiés ou non, émanant des établissements d'enseignement et de recherche français ou étrangers, des laboratoires publics ou privés. 
This is the accepted version of the following article:

Ruiz, M. J., Dojat, M., \& Hupé, J. M. (2020). Multivariate pattern analysis of fMRI data for imaginary and real colours in grapheme-colour synaesthesia. European Journal of Neuroscience, 52(5), 3434-3456,

which has been published in final form at

[https://onlinelibrary.wiley.com/doi/epdf/10.1111/ejn.14774]. This article may be used for non-commercial purposes in accordance with the Wiley Self-Archiving Policy [http://www.wileyauthors.com/self-archiving].

\section{Research Report}

\section{Multivariate pattern analysis of fMRI data for imaginary and real colours}

\section{in grapheme-colour synaesthesia}

\section{Mathieu J. Ruiz (1,2), Michel Dojat (2), Jean-Michel Hupé (1)}

(1) Centre de Recherche Cerveau et Cognition, Université de Toulouse Paul Sabatier \& CNRS, 31300 Toulouse, France

(2) Grenoble Institut des Neurosciences, Université Grenoble Alpes, INSERM \& CHU Grenoble Alpes, 38000 Grenoble, France

Corresponding Author: Jean-Michel Hupé

email: jean-michel.hupe@cnrs.fr

phone: $+33(0) 562746124$

postal address: CNRS CERCO UMR 5549, Pavillon Baudot, CHU Purpan, BP 25202, 31052

Toulouse Cedex 3

Running title: MVPA of grapheme-colour synaesthesia

Keywords: synesthesia; mental imagery; color perception; human vision 


\begin{abstract}
Grapheme-colour synaesthesia is a subjective phenomenon related to perception and imagination, in which some people involuntarily but systematically associate specific, idiosyncratic colours to achromatic letters or digits. Its investigation is relevant to unravel the neural correlates of colour perception in isolation from low-level neural processing of spectral components, as well as the neural correlates of imagination by being able to reliably trigger imaginary colour experiences. However, functional MRI studies using univariate analyses failed to provide univocal evidence of the activation of the 'colour network' by synaesthesia. Applying Multivariate (multivoxel) Pattern Analysis (MVPA) on 20 synaesthetes and 20 control participants, we tested whether the neural processing of real colours (concentric rings) and synaesthetic colours (black graphemes) shared patterns of activations. Region of interest analyses in retinotopically and anatomically defined visual areas revealed neither evidence of shared circuits for real and synaesthetic colour processing, nor processing difference between synaesthetes and controls. We also found no correlation with individual experiences, characterised by measuring the strength of synaesthetic associations. The whole brain, searchlight, analysis led to similar results. We conclude that revealing the neural coding of the synaesthetic experience of colours is a hard task which requires the improvement of our current methodology: e.g. involving more individuals and achieving higher MR signal to noise ratio and spatial resolution. So far, we have not found any evidence of the involvement of the cortical colour network in the subjective experience of synaesthetic colours.
\end{abstract}




\section{Introduction}

Synaesthesia is a subjective experience shared by only a fraction of the population (Simner et al., 2006; Chun \& Hupé, 2013; Simner \& Carmichael, 2015; Rouw \& Scholte, 2016; Watson et al., 2017), offering, in principle, an opportunity to study the neural bases of subjective experience, drawing on individual differences just like in neuropsychology, but involving healthy people. Moreover, colour, the typical prototype of a qualia (what it feels like to perceive something) is the most often cited (or at least studied: Ward, 2013) content of the synaesthetic experience. However, the very subjective nature of the synaesthetic experience represents a major obstacle when trying to set an objective and operational definition, as required in an experimental protocol. Not only subjective descriptions may vary a lot between subjects (Flournoy, 1893), but also within subjects when asked to complete the same questionnaire again (Edquist, Rich, Brinkman, \& Mattingley, 2006) or when describing their subjective experience of colour for different letters (Hupé, Bordier, \& Dojat, 2012b). Using psychophysical tests, the synaesthetic experience of colour appears more similar to imagined or remembered than perceived colours (Witthoft \& Winawer, 2013; Chiou \& Rich, 2014; Hupé \& Dojat, 2015; Janik McErlean \& Banissy, 2017; Chiou et al., 2018). The experience of synaesthetic colours can be indeed formally described as a form of mental imagery, since it occurs without any corresponding spectral stimulation. The obligatory experience of colour when exposed to letters or digits may therefore justify the label of 'intrusive visual imagery' (Reeder, 2016). Unfortunately, this simplification does not help much with defining the phenomenological content of synaesthesia, since self-reports of mental imagery show at least as much diversity as those of synaesthesia (Galton, 1880), with mixed evidence about whether the presence of synaesthesia may relate to individual differences in mental imagery (Chun \& Hupé, 2016). One may, however, study how much synaesthesia requires the neural resources involved in visual perception. This bottom-up approach, which does not address the 
phenomenological issue, can at least be operationalized. Moreover, grapheme-colour synaesthesia offers a unique opportunity regarding the neural correlates of imagination as it restrains both individual variability and the content specificity of visual imagery. Last but not least, synaesthetic colours are systematically triggered by letters and digits, unlike "regular" mental imagery that depends on both the good will and the (uneven) ability of subjects.

Several brain imaging studies, listed and summarized by Hupé and Dojat (2015), have compared activations in the visual cortex for real and synaesthetic colours. The majority of these studies did not reveal any overlap of activation. Moreover, there were questions whether activations triggered by synaesthetic stimuli, when observed, were in fact related to the synaesthetic experience at all (Hupé \& Dojat, 2015). This surprising 'Null' result may be due to methodological limitations since only massive univariate analysis of brain imaging data were used so far, which may reveal only processes well and identically localized in the brains of many subjects (Hupé et al., 2012b). Moreover, "activations" are defined by a subtraction method involving the problematic definition of a baseline or control stimulus and of the statistical procedure used (Hupé, 2015; Shifferman, 2015). Multivariate (multivoxel) Pattern Analysis (MVPA) does not require any subtraction and may identify neural networks distributed within a brain region, possibly differently across subjects (no need for anatomical normalization and averaging). MVPA provides therefore a powerful way to reveal whether specific information is encoded in different brain regions (Cox \& Savoy, 2003; Norman, Polyn, Detre, \& Haxby, 2006; Formisano \& Kriegeskorte, 2012; Hebart \& Baker, 2017). It has been applied successfully to the decoding of aspects of mental images (Thirion et al., 2006; Stokes et al., 2009; Reddy, Tsuchiya, \& Serre, 2010). Using fMRI, here we simply asked whether classifiers trained on patterns of blood oxygenation dependent signals (BOLD responses) elicited by different coloured stimuli could predict which synaesthetic colours were experienced by synaesthetes when seeing achromatic letters and digits. We studied in 
particular the early stages of visual processing by identifying cortical areas V1 to V4 in each subject, using retinotopic mapping, thus avoiding the problems related to structural normalization (Poldrack, 2007; Hupé, 2015). We also explored the whole visual cortex (including parts of the parietal cortex) using regions of interest based on a probabilistic atlas (Eickhoff et al., 2007), and performed whole brain searchlight analyses (Kriegeskorte, Goebel, \& Bandettini, 2006). We compared all the measures obtained in synaesthetes with those obtained in a group of non-synaesthetes to take into account any potential non-specific effect related to the choice of stimuli. We also took into account the individual variability of the synaesthetic experience: without any possibility to characterize objectively the different phenomenological accounts, we measured the strength of the synaesthetic associations (Ruiz \& Hupé, 2015).

\section{Materials and Methods}

\section{Participants}

We tested 20 synaesthetes and 20 non-synaesthetes. Since this study was the first of the kind and therefore exploratory, group size was decided arbitrarily when submitting the protocol to the ethics committee, based on the published study using the most similar protocol (Bannert \& Bartels, 2013, who tested 18 subjects: see Discussion) and feasibility considerations.

Synaesthetes (17 women) were between 21 and 42 years old $(\mathrm{M}=27.9, \mathrm{SD}=5.5)$.

Recruitment was diverse and opportunistic, based on self-referral following publicity on internet: lab webpage, Facebook event, announcements on university networks in Grenoble and Paris. Potential participants, after a first phone interview, were asked by email to fill-up a questionnaire to describe their synaesthetic associations and for grapheme colour associations to send us a list of those. Synaesthetes were included if they had a sufficient and diverse 
number of letter-colour and digit-colour associations as required by the design of our experiments (see below). When they came to the laboratory to perform the experiments, they had a semi-directed interview to evaluate the phenomenology of their synaesthetic associations. They also ran a modified version of the "Synaesthesia Battery Test" (Eagleman, Kagan, Nelson, Sagaram, \& Sarma, 2007) to choose precisely the colour of each letter and digit: each letter or digit was presented in a random order and with a random colour on a computer screen over a grey background. Synaesthetes had to adjust the hue, saturation and luminance of the grapheme by using a colour palette presented on the side of the grapheme (see an example of a screen capture page 141, Figure 6.1.1 of Ruiz's thesis, 2014; see page 158, Figure 6.4.6, for the version adapted to the scanner environment). Subjects could indicate if the grapheme had no synaesthetic colour; they could choose to see the grapheme printed in lower or upper case; they could display a square patch instead of the grapheme; they could also momentarily hide the colour palette. All these options were designed to take into account the diversity of phenomenological experience of synaesthetic colours. Synaesthetes took as long as they wished.

This procedure was also used as a retest to confirm the validity of the first-person reports (Ruiz \& Hupé, 2015): in all subjects, all chosen colours matched those indicated by print or by name in the questionnaire. In addition, objective measurements of synaesthetic associations were obtained by Stroop-like tests (see below in the 'Protocol' section). Seven of the included synaesthetes had already participated in psychophysics experiments between 2007 and 2010 (Ruiz \& Hupé, 2015).

Control participants were recruited after synaesthetes to match their demographic statistics (16 women, age range between 23 and 38 years old, $\mathrm{M}=28.5, \mathrm{SD}=4.3$ ), following similar advertisement strategies as well as soliciting colleagues at the Grenoble Institute of Neuroscience. Interviews were conducted to verify the absence of any type of synaesthesia, 
not only the absence of grapheme-colour associations. We chose not to run any consistency score with the control subjects in order not to prime them to do any voluntary association between graphemes and colours before the tests in the scanner. It could be argued that some of the controls may have had implicit synaesthetic associations they were not aware of, as it sometimes happens. In any case, this unlikely possibility could not bias our results because most of our analyses did not require any direct comparison of the performances by synaesthetes and controls.

The study was performed in accordance with the World Medical Association Declaration of Helsinki, it received approval by the Institutional Review Board of Grenoble (CPP 12-CHUG17, approval date 04/04/2012) and written, informed consent was obtained from all subjects. A medical doctor verified that all subjects were without past or current brain disease and had no detected cognitive deficit. All subjects had normal colour perception on the Lanthony D-15 desaturated colour test (Richmond products), and normal or corrected to normal eyesight (then using MRI-compatible glasses).

\section{Materials}

Stimuli: for each synaesthete, we tried to identify four pairs of graphemes made of one letter and one digit that had similar colour associations. We never chose graphemes for which a synaesthete indicated several colours. We tried to find pairs of red, green, blue and yellow ( $R$, G, B, Y) graphemes, but we were only partially successful and in some cases we selected a pair from the most saturated colours available. Figure 1 shows the actual letters and digits with colours used in the experiments. Only 13 subjects named the pairs red, green, blue and yellow; other colours were named orange, violet, fuchsia and brown, as well as light and dark blue or green. Syn08 and syn48 had a pair made of two letters. Since each synaesthete was tested with a different set of stimuli, each control subject was tested with the stimuli of a specific synaesthete (with the exception of syn10 who had no matched control, by mistake; 
two controls were tested instead with the stimuli of syn11. Paired comparisons were therefore based on 38 subjects).

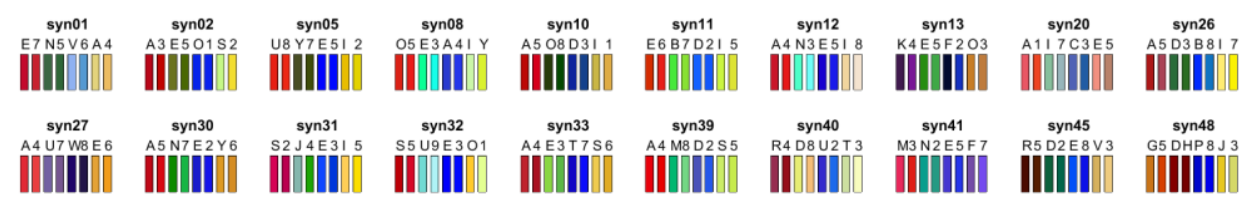

Figure 1. Letters and digits used for each synaesthete, with their corresponding synaesthetic RGB colours (the rendering of the colours using the projector in the scanner was different).

In the MR scanner, we presented these letters and digits in black at the centre of the screen (upper case, Helvetica font, extent up to 2 degrees eccentricity) over a grey uniform background (CIE xyY [0.29 0.3 77.4], half of the maximal luminance of the screen). Stimuli were projected on a translucent screen at the back of the scanner by a video projector Epson EMP 8200. We used a spectrophotometer (PhotoResearch PR 650) for colour and luminance measurements used to compute calibrated images. We also presented dynamic concentric rings (square luminance profile, similar to the stimuli used by Brouwer \& Heeger, 2009, except for the absence of anti-aliasing so as to use only the colours selected by each synaesthete), with the exact same (real) colours as those chosen by each synaesthete for each grapheme. The choice of colours matching the individual grapheme ' $R, G, B, Y$ ' colour associations was done again by each synaesthete in the scanner over the same grey background, using a house-modified MRI compatible, comfortable, 10-button console controller, and the colour-picker of the "Synaesthesia Battery Test" as was done previously outside of the scanner. The same coloured rings were used for each matched control. The rings extent was also up to 2 degree eccentricity and the spatial frequency was 3 cycles/degree (six circles). The phase of the rings changed randomly at $6 \mathrm{~Hz}$ to almost nullify visual effects induced by the absence of anti-aliasing. 
These stimuli were chosen with the purpose of training and testing classifiers (see below, "Data analysis: classifications"). Briefly, we wanted to use the BOLD responses to the coloured rings to train classifiers on colours (four categories), and the BOLD responses to graphemes to train classifiers on synaesthetic colours (same four categories). This required choosing pairs of dissimilar graphemes, i.e. a letter and a digit, to try to avoid that the classifier trained on some shared form features, but rather on their common associated colour. This also implied that decoding should not be feasible that way based on the responses of control subjects. The use of pairs of graphemes also allowed the training on letters and testing on digits (or the reverse), with success in principle possible only for synaesthetes, based on their synaesthetic colour associations. The careful matching procedure of synaesthetic colours allowed the training of classifiers on real colours and testing on graphemes to identify which brain regions, if any, coded both real and synaesthetic colours. Again, any decoding success would in principle be possible only in synaesthetes.

Classifiers would be trained and tested on four categories, 'R, G, B, and $Y^{\prime}$, referring either to the real or the synaesthetic prototypical colours that we tried to select. To maximize classification performances, categories should be perceptually disjoint. Figure 2 represents the actual colours used in the scanner for each synaesthete within the CIE L*a*b* colour space, which is more perceptually uniform than the CIE xyY space. As was already obvious in Figure 1, differences of luminance, in addition to differences in hue, were important to distinguish stimuli. Figure 2 illustrates that the hue and luminance distances were not similar across subjects between categories and within pairs, leading to unequal clusterisation. We could even expect some confusions by the classifiers for some subjects (e.g. "green/yellow" for syn11, "red/blue" for syn13 or "blue/yellow" for syn41). While the maximal theoretical performance achievable by classifiers was therefore below $100 \%$, classifiers could however obtain more than the $25 \%$ chance performance in every subject. 
To fit with the synaesthetic individual experience, stimuli were not isoluminant. This constitute a major difference with other MVPA studies of the neural correlates of colour processing, which used isoluminant stimuli (Brouwer \& Heeger, 2009; Parkes, Marsman, Oxley, Goulermas, \& Wuerger, 2009). At the cortical level, the visual circuits rely on both (but with different degrees) the parvo- and the magno-cellular pathways (Tootell \& Nasr, 2017). One could therefore question whether colour classification would be really based on colours and not just luminance. In fact, classifiers could not rely on luminance only, because many pairs of different colours had a similar luminance (same size of the crosses on Figure 2). Figure 2 reveals that at least 22 distinctions of pairs of stimuli could not be achieved by classifiers using only luminance cues (classification followed a one-versus-one heuristic, see below): for example "blue/yellow" and "red/blue" for syn01. Seven distinctions only would be difficult for classifier using only hue differences (for example "green/yellow" for syn02, where the distance between one green and the yellow crosses is smaller than the distance between the two yellow crosses). In order to quantify systematically the relative weight of luminance and hue information in our set of stimuli, we computed a measure of colour clusterisation: the average geometric distance in the $\mathrm{L}^{*} \mathrm{a} * \mathrm{~b}$ space between the average of colour pairs divided by the average geometric distance within colour pairs (the resulting “Distance ratio' measure is indicated for each subject in Table 1). Then we computed again this measure using either only the ' $L$ ' luminance component or the ' $a$ ' and ' $b$ ' hue components. The $\mathrm{L}^{*} \mathrm{a} * \mathrm{~b}$ Distance ratio was highly correlated to the ' $\mathrm{a} * \mathrm{~b}$ ' Distance ratio (nonparametric Spearman $R=0.98)$, not to the ' $L$ ' Distance ratio $(R=0.4, P=0.078)$.

We obtained a further, indirect but suggestive, indication that the luminance component was not the main feature used for stimulus classification, by taking advantage of the different values of the Distance ratio across subjects. We speculated that the colour classification ('Col' classification, based on coloured rings stimuli - see below) could be easier when this ratio 
was higher. We performed a searchlight correlation analysis across participants between the Distance ratio and the performance of the 'Col' classifier. When testing the group of synaesthetes (no cluster reached significance in the group of controls), we found one significant cluster $\left(P<0.001\right.$ uncorrected voxel level, $P_{F W E}<0.001$ cluster level, 106 voxels, $2862 \mathrm{~mm}^{3}$ ), precisely in the left fusiform gyrus, peaking at MNI XYZ $=[-27-73-4]$, extending from about V4 to FG4, in line with the involvement of this region in colour processing. Importantly, the correlation analysis based on hue only ( $a * b$ Distance ratio) revealed the same cluster, but not the correlation analysis based on luminance only (L Distance ratio). For all the other analyses with the other classifiers (described below), we did not find any correlation between the performance of classifiers across subjects and the index of colour Distance ratio.
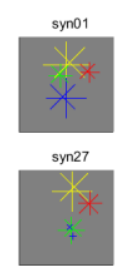

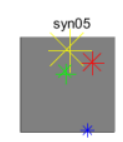

syn31
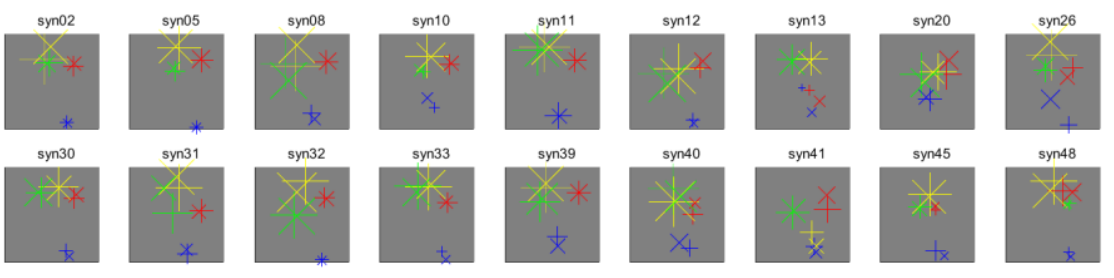

Figure 2. Colour coordinates in the CIE L*a*b* space of the stimuli used for each synaesthete, corresponding to the idiosyncratic synaesthetic colours of letters (+) and digits (x). The colours of the crosses are arbitrary and correspond to the four categories the classifiers had to distinguish. The size of the crosses is proportional to luminance (marker size $=0.4 * \mathrm{~L}$, where $\max (\mathrm{L})=100$; axes limits are $+/$ 130 , possible range being -128 to +127$)$.

\section{Protocol}

Each subject ran three fMRI sessions of about 1 hour. In addition, synaesthetes ran a 1 hour psychophysics experiment (before or interleaved with fMRI experiments, depending on schedule availability) to measure the strength of their synaesthetic associations using variants of Stroop tasks. 
All the details of the psychophysics experiment as well as the psychophysics results of 11 synaesthetes are published (Ruiz \& Hupé, 2015). Briefly, eight graphemes (repeated 36 times each) were presented randomly either with the colour chosen by each synaesthete (congruent condition) or with the synaesthetic colours of the other presented graphemes (incongruent conditions). Synaesthete had to name as fast as possible the real colour of the grapheme ('colour' task). Response times were measured a posteriori based on the audio recording. The procedure was then repeated, but synaesthetes had this time to name as fast of possible the name of the synaesthetic colour (called a photism) they associated to each grapheme, which was also either congruent or incongruent with the real colour of the stimulus ('photism' task). The index of the strength of synaesthetic associations ('Photism strength') combined two measures: the response time difference for congruent and incongruent stimuli in the colour task, which reflects the difficulty to inhibit synaesthetic associations; the response time difference to name the real and the synaesthetic colours (in the congruent condition), which reflects how easily synaesthetes retrieve the synaesthetic colour.

The data of one synaesthete (syn40) could not be analysed because the chosen orange and yellow/green colours revealed too similar (see Figure 2) and were not named consistently over the course of the experiment. Table 1 provides a summary of the data. It shows that even synaesthetes who obtained a relatively low score of Photism strength (e.g., syn01) were very fast at naming the synaesthetic colour of letters and numbers, even though the real colour of the stimulus was systematically varied. Moreover, they very rarely made any mistake (Ruiz \& Hupé, 2015). Such a task would be extremely difficult to perform by any non-synaesthete trying to memorize (without training) random colour associations. These data therefore provide a further objective validation of the genuineness of the synaesthetic experience of these participants as well as an estimate of the strength of the associations. 
Table 1. Demographics and characteristics of the tested synaesthetes.

\begin{tabular}{|c|c|c|c|c|c|c|c|c|c|c|}
\hline & & & & & Colour & & & Photism & & \\
\hline & & & & & $\begin{array}{c}\text { Congruent } \\
(\mathrm{ms})\end{array}$ & $\begin{array}{c}\text { Incongruent } \\
(\mathrm{ms})\end{array}$ & $\begin{array}{l}\text { Congruency } \\
\text { effect (ms) }\end{array}$ & $\begin{array}{c}\text { Congruent } \\
(\mathrm{ms})\end{array}$ & $\begin{array}{c}\text { Incongruent } \\
(\mathrm{ms})\end{array}$ & $\begin{array}{c}\text { Photism } \\
\text { delay }(\mathrm{ms})\end{array}$ \\
\hline Synaesthete & Age & Sex & $\begin{array}{l}\text { Distance } \\
\text { ratio }\end{array}$ & $\begin{array}{c}\text { Photism } \\
\text { strength } \\
\text { (ms) }\end{array}$ & $\mathrm{CC}$ & CI & CI-CC & $\mathrm{PC}$ & PI & PC-CC \\
\hline syn01 & 21 & $\mathrm{~F}$ & 13 & -107 & 443 & 454 & 12 & 561 & 685 & 119 \\
\hline syn02 & 21 & $\mathrm{~F}$ & 13 & 52 & 466 & 543 & 77 & 491 & 543 & 25 \\
\hline syn05 & 30 & F & 38 & 20 & 452 & 501 & 49 & 480 & 568 & 29 \\
\hline syn08 & 35 & M & 6 & 69 & 484 & 585 & 101 & 516 & 619 & 32 \\
\hline syn 10 & 31 & $\mathrm{~F}$ & 9 & 40 & 556 & 633 & 77 & 593 & 689 & 37 \\
\hline syn11 & 28 & F & 21 & 146 & 643 & 782 & 138 & 636 & 681 & -7 \\
\hline syn 12 & 33 & $\mathrm{~F}$ & 9 & 136 & 597 & 683 & 86 & 548 & 646 & -50 \\
\hline syn13 & 29 & F & 4 & 30 & 668 & 744 & 76 & 714 & 795 & 46 \\
\hline syn 20 & 23 & $\mathrm{~F}$ & 4 & 19 & 506 & 556 & 50 & 537 & 637 & 31 \\
\hline syn26 & 27 & M & 4 & -19 & 497 & 528 & 31 & 547 & 690 & 50 \\
\hline syn 27 & 24 & $\mathrm{~F}$ & 10 & -4 & 547 & 582 & 36 & 586 & 676 & 39 \\
\hline syn30 & 27 & $\mathrm{~F}$ & 14 & 416 & 466 & 844 & 378 & 427 & 449 & -39 \\
\hline syn31 & 24 & F & 6 & 20 & 525 & 573 & 47 & 553 & 639 & 27 \\
\hline syn32 & 33 & $\mathrm{~F}$ & 12 & -57 & 456 & 477 & 21 & 534 & 620 & 78 \\
\hline syn33 & 30 & F & 11 & -30 & 447 & 457 & 10 & 487 & 525 & 40 \\
\hline syn39 & 26 & F & 13 & 48 & 440 & 523 & 83 & 475 & 582 & 35 \\
\hline syn40 & 42 & F & 5 & $\mathrm{NaN}$ & & & & & & \\
\hline syn41 & 23 & $\mathrm{~F}$ & 6 & -15 & 484 & 514 & 30 & 529 & 678 & 45 \\
\hline syn45 & 27 & F & 10 & 10 & 539 & 564 & 25 & 554 & 691 & 16 \\
\hline syn48 & 22 & $\mathrm{M}$ & 9 & -73 & 487 & 529 & 42 & 602 & 770 & 115 \\
\hline
\end{tabular}

The variable 'Distance ratio' is a measure of clusterisation of the pairs of colour (average betweencluster distance divided by average within-cluster distance, measured in the $\mathrm{L}^{*} \mathrm{a} * \mathrm{~b} *$ space). The higher the value, the better the clusterisation (see Figure 2). The variable 'Photism strength' is the measure of the strength of synaesthetic associations as developed by Ruiz et al. (2015), based on the results of Stroop like tests. The next columns indicate the median time measured in $m s$ for each subject to name either the real ('Colour') or the synaesthetic colour ('Photism') of the letters and numbers shown in Figure 1, when the real colour was either congruent or incongruent with the synaesthetic colour indicated by each synaesthete. The 'Congruency effect' was measured as the difference of response time in the 'Colour' condition. 'Photism delay' is the difference between naming the real and the synaesthetic colours, and 'Photism strength' is the difference of these two values. The interpretation of this index is only relative (the zero value does not have any special meaning). The data of syn40 were not consistent (see text). 


\section{fMRI experiments}

The MR experiments were performed at the IRMaGe MRI facility (Grenoble, France) with a 3T Philips Intera Achieva, using a 32 channels coil. The experiments can be decomposed successively in three "sessions" (about 1 hour each), "runs" (a few minutes), "blocks" (1 minute) and "events" (1 second). One session was dedicated to retinotopic mapping and functional localizer runs using pictures of objects, words and coloured stimuli (Mondrian). These latter runs were included to test whether voxels involved in the decoding of synaesthetic colours were located in regions well-defined functionally, respectively the Lateral Occipital Complex (LOC: Grill-Spector, Kourtzi, \& Kanwisher, 2001), the Visual Word Form Area (VWFA: Dehaene \& Cohen, 2011) and “colour centres” (Hupé et al., 2012b). We did not have the opportunity to use those localizers (see Results). Retinotopic mapping was performed strictly as described in a previous study (Bordier, Hupé, \& Dojat, 2015), using the Brain Voyager analysis pipeline to define in each subject the ventral and dorsal as well as the left and right parts of areas V1, V2, V3 and V4 (ventral only). The parameters of the EPI functional images were TR/TE: 2000/30 ms, excitation pulse angle: $80^{\circ}$, acquisition matrix: 80x80, bandwidth: $54.3 \mathrm{~Hz} /$ pixel, isotropic nominal resolution: $3 \mathrm{~mm}$, $30 * 2.75 \mathrm{~mm}$ thick slices with $0.25 \mathrm{~mm}$ interspace covering the whole visual cortex, with four additional dummy scans. To allow the precise alignment of functional scans across sessions, a high-resolution structural image of the brain was also acquired using a T1-weighted MPRAGE sequence. The sequence parameters were TR/TE: 25/2.3 ms, excitation pulse angle: $9^{\circ}, 180$ sagittal slices of $256 * 240$ (read x phase), bandwidth: $542.5 \mathrm{~Hz} /$ pixel isotropic nominal resolution: $1 \mathrm{~mm}$, for a total measurement time of $4 \mathrm{~min} 31 \mathrm{~s}$. 
Another session was dedicated to the "synaesthesia" protocol (a structural image was also acquired with the same parameters as in the first session, in the middle of the functional runs). Twelve functional runs were acquired. The parameters of the EPI functional images were identical to those used for the retinotopic mapping experiment but TR: $2500 \mathrm{~ms}$ for an acquisition volume of 45 slices covering the entire brain with a total measurement time of 3 min $47.5 \mathrm{~s}$ (87 images including the four dummy scans as well as 3 additional images at the end of the run to account for the random intervals between stimuli). In each functional run, stimuli of one type only were presented: letters, digits, concentric rings with the synaesthetic colours of letters, or concentric rings with the synaesthetic colours of digits. The session contained three successive sequences of four runs, each run with a different stimulus type (with a different random order of stimulus type in each sequence). Each run contained $3 * 60 \mathrm{~s}$ blocks of a rapid-event paradigm, followed by 10 s fixations. Stimuli of different "colours" were presented pseudo-randomly in each block to optimize the estimation of the main effects (the sequence of the events was designed so as to optimize the efficiency of the estimation both of the main and of the differential effects: Friston, Zarahn, Josephs, Henson, \& Dale, 1999. Random sequences were generated and the sequence producing the better estimation was selected). For example, in a letter block for syn01 and her matched control, the letters E, $\mathrm{N}, \mathrm{V}$ and $\mathrm{A}$ were presented six times each for $1 \mathrm{~s}$, with $1 \mathrm{~s}+/-333 \mathrm{~ms}$ fixation only between each letter (each block lasted therefore only approximately 60s). This protocol allowed an estimation of the BOLD response to each letter in each block (beta weights, using a General Linear Model, see below) based on six presentations. We obtained three estimations (betas) in each run for each "colour", for a total of thirty-six estimates ( $9 * 4$ "colours") for each type of stimulus to be used by classifiers. The power of classification algorithms depends on both the number and quality (signal to noise ratio) of estimates (called exemplars). The present compromise between quantity and quality was based on (Mumford, Turner, Ashby, \& 
Poldrack, 2012) and on preliminary experiments (Ruiz, Hupé, \& Dojat, 2012). Subjects had to fixate the centre of the screen (the fixation point, present between stimuli and at the centre of the coloured rings, or the centre of the grapheme) and pay attention to the stimuli for the whole duration of each run. To help subjects maintain attention, they performed a one-back task (pressing a button each time the same stimulus was repeated twice in a row).

In the remaining session, a high-resolution, high-contrast structural image of the brain was acquired using a T1-weighted MP-RAGE sequence. The sequence parameters were TR/TE/TI: $25 / 3.7 / 800 \mathrm{~ms}$, excitation pulse angle: $15^{\circ}$, acquisition matrix: 180 sagittal slices of 256*240 (read x phase), bandwidth: $191 \mathrm{~Hz} /$ pixel, readout in anterio-posterior direction, number of averages: 1 , sense factor anterio-posterior: 2.2 , right-left: 2 , isotropic nominal resolution: $1 \mathrm{~mm}$, with a total measurement time of 9 min $41 \mathrm{~s}$. This image was the structural reference image of each subject. We also acquired diffusion-weighted images, analysed in another study (Dojat, Pizzagalli, \& Hupé, 2018) and a sequence of functional resting state (not analysed yet).

We recorded oculomotor signals during the scans with an ASL EyeTracker 6000. At the beginning of each session, subjects had to fixate each point of a calibration matrix, and were therefore aware that the quality of their fixation was monitored. However, signal quality in some subjects was not good enough or not constant, or even too poor to be of any use for subjects who had to wear non-magnetic glasses in the scanner, so we did not even attempt to analyse these data. We can only speculate that subjects had a better fixation than if they did not know that their gaze was recorded. Whole brain univariate analyses did not reveal any activation along the anterior calcarine and the parieto-occipital sulcus, where activations correspond to the signature of blinks (Hupé, Bordier, \& Dojat, 2012a), providing indirect evidence that the distributions of blinks were not correlated with our stimuli presented randomly. 


\section{Data Analysis}

The standard pre-processing procedure of functional images was applied using SPM8: slicetiming correction, then motion correction with realignment, together with correction of spatial distortions of the static magnetic field (Vasseur et al., 2010). The within session structural image was realigned to the mean EPI image, as well as the high resolution high contrast structural image, but no further transformation of the EPI images was performed. No spatial smoothing was applied for MVPA, as maximally differential activation of voxels was shown to maximize the power of classifiers (Ruiz et al., 2012). This was confirmed on these data when testing spatial filters with FWHM $=3,6$ and $9 \mathrm{~mm}$. Transformation matrices were computed between the structural image and the MNI template to allow the transformation and projection of atlas-based masks of specific anatomical structures (Anatomy Toolbox for SPM8 Version 2.2b, 2016) into the subject's space.

For MVPA (our main analysis), for each subject and each run we first ran a General Linear Model (GLM). The six parameters of motion correction were included as factors of noninterest in the design matrix. Thirteen main predictors, four events (grapheme or colour) * three blocks plus one for when only the fixation point was shown, were obtained by convolving the canonical HRF with Dirac functions corresponding to the time of presentation of each stimulus (the fixation period was modelled as a null event whereas the GLM intercept models the session effect). The corresponding beta weights estimated by the GLM for each colour (real or synaesthetic) and stimulus type (ring or grapheme), divided by the square root of residuals (in order to scale the signals, as requested for pattern classification algorithms: Misaki, Kim, Bandettini, \& Kriegeskorte, 2010; Mumford et al., 2012), were used as examples by a Support Vector Classification (SVC) algorithm (Scikit-learn version 0.15.2, implemented in Python version 2.7.9.0: Pedregosa et al., 2011). We used a linear kernel (default value of the $\mathrm{C}$ parameter $=1$ ) and a one-versus-one classification heuristic to classify 
each example in one of the four categories. For all five classifications described below, training and test runs were always fully independent: betas obtained from blocks from the same run were never split between training and test runs.

Classifications. We trained and tested five families of classifiers (Figure 3). Six runs (eighteen blocks) were used for colour ('Col' family of classifiers) and synaesthesia ('Syn') decoding. The procedure was leave-one-run-out. Six classifiers were therefore trained to classify $(5$ runs $* 3$ blocks $* 4$ colours $=60)$ colour exemplars in four categories, and tested on $(1$ run $* 3$ blocks $* 4$ colours $=12)$ independent exemplars. Performance was therefore computed over seventy-two classifications ( 6 classifiers * 12 tested exemplars), with chance level $=25 \%$. For performance at the chance level, $95 \%$ of the individual scores should be within $11(15 \%)$ and 25 (35\%) successful classifications over 72 (binomial distribution). For grapheme runs, training was performed on pairs made of one letter and one digit. If the decoder learnt only the letters, for example (by being able to filter out the responses to digits), then performance on decoding letters and digits could reach up to 50\%, without knowing anything about synaesthetic colours. One could expect, however, that classification performance for synaesthetes would be higher than for controls because of the additional information provided by synaesthetic colours. A more stringent test of synaesthetic coding ('g1g2') was the training of one classifier on letters (3 runs * 12 exemplars) and testing on digits (and the reverse). Learning was achieved using thirty-six exemplars (letters or digits) to be classified in four categories, test was on thirty-six exemplars (digits or letters), for a total performance over seventy-two classifications by combining training on letters and training on digits. To evaluate if brain regions coded both real and synaesthetic colours (' $\mathrm{C} 2 \mathrm{~S}$ '), training was performed by one classifier on six colour runs (seventy-two exemplars), test on six grapheme runs (seventy-two exemplars). We also performed the reverse classification ('S2C'). 


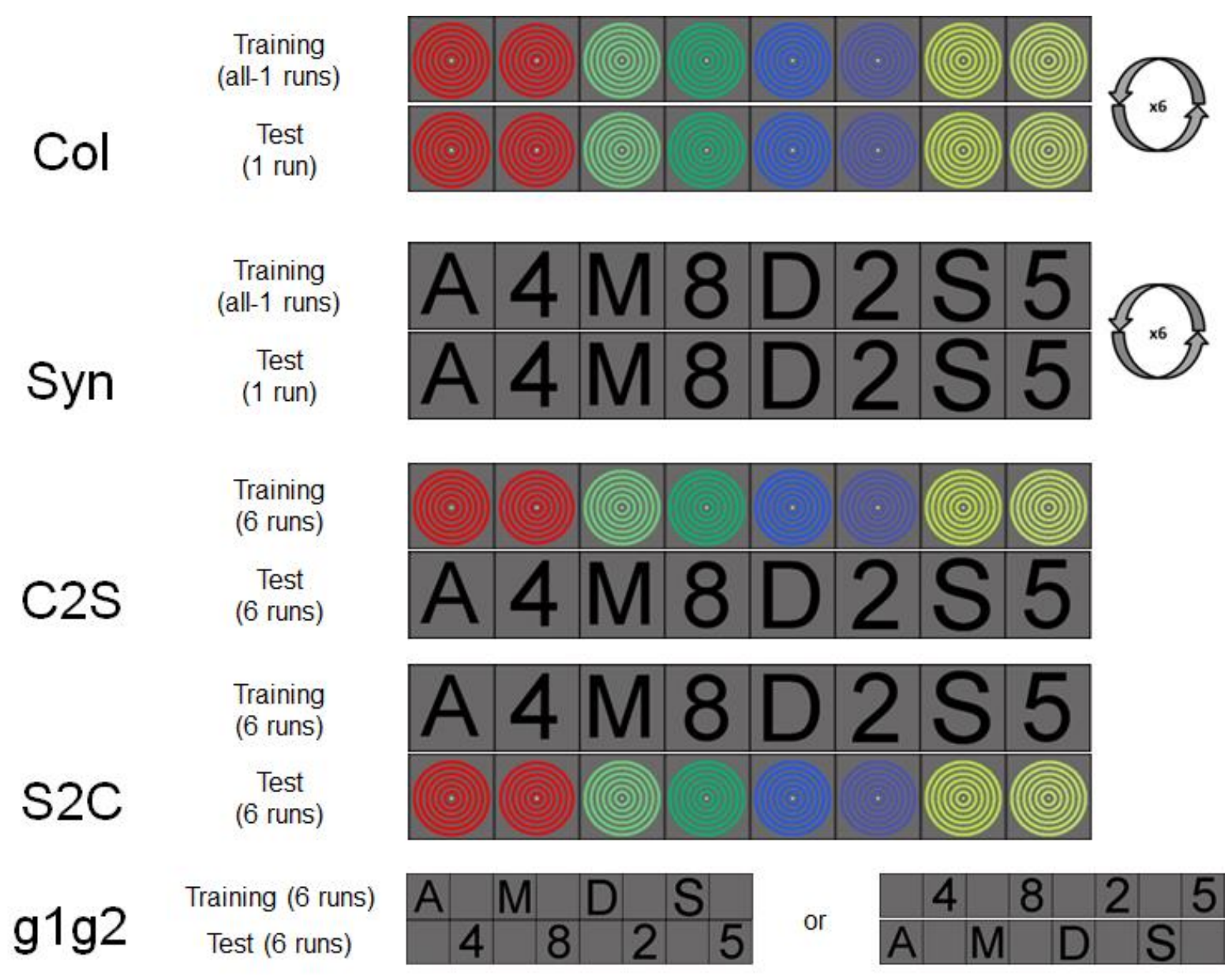

Figure 3. MVPA classifications. 'Col' classification: The procedure was leave-one-run-out. Six classifiers were trained to classify 60 colour exemplars from 5 runs in four categories, and tested on 12 independent exemplars of the remaining run. Performance was therefore averaged over seventy-two classifications ( 6 classifiers * 12 tested exemplars). 'Syn' classification: The procedure was the same as for the 'Col' classification, based on pairs of graphemes and therefore also synaesthetic colours for synaesthetes. 'C2S' classification: Training was performed by one classifier on six colour runs (seventy-two exemplars), test on six grapheme runs (seventy-two classifications). 'S2C' classification: The procedure was the same as for the ' $\mathrm{C} 2 \mathrm{~S}$ ' classification. ' $\mathrm{g} 1 \mathrm{~g} 2$ ' classification: Classifiers were trained on letters ( 3 runs * 12 exemplars) or digits ( 3 runs) and tested respectively on digits or letters. Overall performance was based on seventy-two classifications.

We computed MVPA in regions of interest (ROIs) defined in each native (non-transformed) subject space. We used visual areas defined by individual retinotopic mapping as well as atlas-based ROIs (Figure 4). We expected synaesthetic colours to involve the ventral visual 
pathway, anterior to V4, so we tested the four subdivisions of the fusiform gyrus (FG, Figure 4a). Some studies have also suggested the role of parietal areas, even though no consensus emerged about exactly which part if any may be involved (Hupé \& Dojat, 2015), so we defined ROIs in parietal regions (Figure $4 b$ ).

a

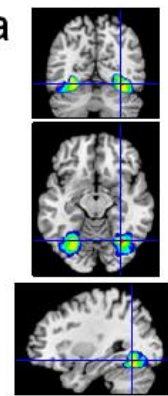

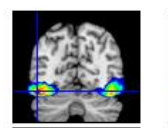
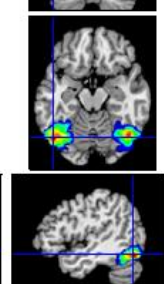
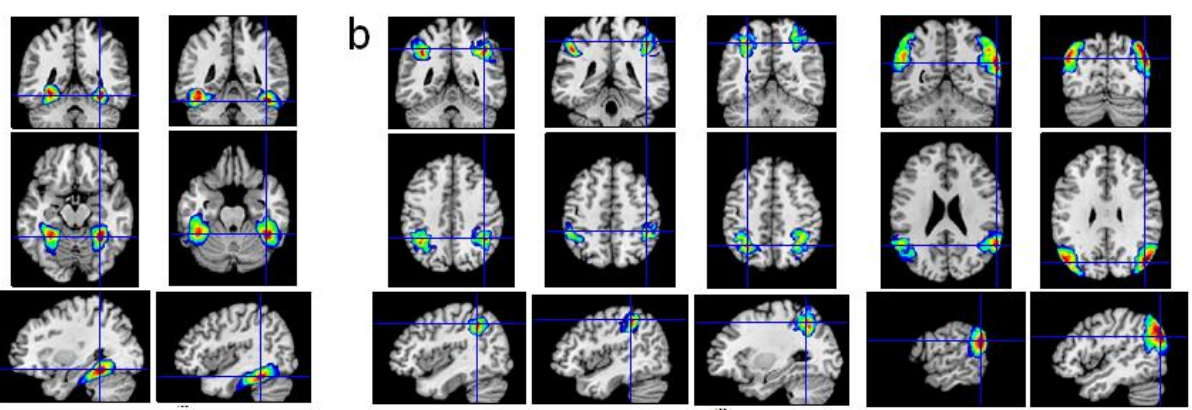

Figure 4. a. Atlas-based regions of interest (ROI) of the fusiform region. From left to right, FG1, FG2, FG3 and FG4. Colour gradients denote the probability of being in the specified ROI, from 0\% (dark blue) to $100 \%$ (dark red). We considered the largest ROI as the mask of the corresponding region. $\mathbf{b}$. Parietal ROIs. From left to right, AIPS_IP1, AIPS_IP2, AIPS_IP3, IPL_PGa and IPL_PGp. See text for full names and references of these areas.

For each subject, anatomical ROIs were defined as the intersection of the subject's grey matter mask and the mask of the anatomical ROIs (Anatomy Toolbox for SPM8: Eickhoff et $a l ., 2007)$ projected into the subject's space. Both retinotopic and atlas-based ROIs had different number of voxels within and across subjects. The performance of classifiers may depend on the number of voxels (called "features" for the algorithm), making difficult the comparison of absolute performance in different ROIs. Between-subject differences may also bias group comparisons.

To address this issue, we first tested ROIs of different sizes by merging retinotopic areas and subdivisions of the fusiform areas and of the parietal areas. The pattern of results were similar whatever our grouping choice of ROIs. We present the results for ROIs of intermediate size (we indicate the min and max number of voxels across subjects in each ROI), combining within single ROIs the right and left parts of retinotopic areas (V1 $=[206441]$, V2 $=[125$ 
420], V3 $=[156$ 340], V4 $=[94$ 268] $)$, the two posterior $($ J. Caspers et al., 2013) $($ left $=$ $\left[98\right.$ 150], right $\left.=\left[\begin{array}{ll}61 & 125\end{array}\right]\right)$ and anterior $($ Lorenz et al., 2017) $($ left $=[174$ 331], right $=$ [127 271]) parts of the fusiform areas, the 3 subdivisions of the Intaparietal Sulcus (Choi et al., 2006; Scheperjans et al., 2008) $($ left $=[197$ 311], right $=[191275])$ and the anterior and posterior parts of the Inferior Parietal Lobule (S. Caspers et al., 2006; S. Caspers et al., 2008) $($ left $=[131335]$, right $=[124279])$.

We also defined ROIs using the same number of voxels in each subject and ROI. To do that, for all classifications, we selected 100 voxels with the highest $F$-scores to colours in each area (we tested different selection sizes and found that 100 was about the optimal number of voxels to reach maximum performance). In order to have enough voxels to choose from in every subject, we selected voxels in only six large areas: the left and right retinotopic areas V1 to V4 (minimum number of voxels across subjects were respectively 352 and 327 ), the left and right fusiform areas FG1 to FG4 (298 and 188) and the left and right parietal areas (347 and 315). Such a selection provides the best chances for colour classifiers (since we select voxels maximally modulated by colours), but classification is then not independent of selection when measuring colour decoding after selection of $F$-scores to colours (but classification is independent for grapheme decoding). In order to provide a fair measure of colour decoding performance to compare grapheme decoding with, voxels were selected using $F$-values computed based only on runs used for training, meaning that each of the six training sets was based on a different set of voxels. For other classifications, the same set of voxels was used based on $F$-values computed across all colour runs.

\section{Statistical analysis}

In each ROI, we computed the mean and $95 \%$ CIs of the performance of classifiers in each group. CIs were computed using a mixed-effect generalized linear model, with a binomial family and a logit link function, as implemented in the library lme4 (Bates, Mächler, Bolker, 
$\&$ Walker, 2015) in $R$, version 3.3.3. CI values were back-transformed from the logit scale using the $R$ function and library lsmeans. Mixed-models provide more accurate estimates than estimates based on second-level statistics (the scores of each subject) because they take into account the number of trials used to compute each score. Here, because each subject performed the same number of trials, second level statistics using one sample $T$-tests (mean +/- 2.09 SEM) produced almost identical CI values (for example, in area V4 and for the ' $\mathrm{C} 2 \mathrm{~S}$ ' classification, the CI plotted in Figure 5 is $[24.8,29.4] \%$, whereas the $T$-test CI is $[24.3$, 29.7]\%).

Formally, 95\% CIs do not allow any inference on population values. They simply indicate that, if we should redo the same experiment many times, $95 \%$ of the $95 \%$ CIs computed that way will include the true population value within their 95\% CI. However, for "well-behaved" distributions like the binomial and normal distributions, as used here, 95\% CIs are almost identical to 95\% Credibility Intervals computed using a flat prior (Kruschke, 2015). For example, in retinotopic $\mathrm{V} 4$, the $95 \%$ credibility interval for the average ' $\mathrm{C} 2 \mathrm{~S}$ ' performance of the 20 synaesthetes is equal to $[24.3,29.8] \%$, meaning that there is $95 \%$ chance that the true ' $\mathrm{C} 2 \mathrm{~S}$ ' decoding performance of $\mathrm{V} 4$ is between $24.3 \%$ and $29.8 \%$ (computations using the $R$ package nimble version 0.8.0: de Valpine, Turek, Paciorek, Anderson-Bergman, Lang, \& Bodik, 2017; the values are exactly the same here for a Bayesian mixed model).

Deciding on the presence or the absence of an effect requires "establishing a region of practical equivalence (ROPE) around the null value that expresses a range of parameter values that are equivalent to the null value for current practical purposes" (Kruschke \& Liddell, 2018). For this study we do not have much information in the literature to decide which values should be considered as equivalent to the $25 \%$ chance level. Every reader would probably consider that $26 \%$ is equivalent to chance in this context. But what about $30 \%$ ? If we had defined a ROPE $=[20,30] \%$, the Bayesian analysis would indicate that $98.3 \%$ of the 
likely values for the mean performance are within the ROPE - meaning that we could state that there is $98.3 \%$ chance that the ' $\mathrm{C} 2 \mathrm{~S}$ ' decoding performance is at chance level in V4 for current practical purposes. For the decoding of real colours, on the other hand, for example in the left retinotopic areas (retL), where the $95 \% \mathrm{CI}$ is $[34,41] \%$ (see Figure 7 ; here the credibility interval is exactly the same), the Bayesian analysis indicates that there is $99.98 \%$ chance that the performance is above the ROPE, so we could be extremely confident that colours can be decoded. Without any definition of the ROPE, our 95\% Confidence Intervals show the range of "true" performance values roughly compatible with our data.

We also computed the mean and $95 \%$ CIs of the between group differences. We performed both independent and paired comparisons. Paired comparisons are in principle more appropriate and powerful with this protocol, because it cancels any difference due to the specific choice of colours and graphemes; however, for voxels not concerned with those small differences, pairing is artificial and may just bring some noise. Results were in any case very similar for both comparisons. We show the 95\% CIs for paired comparisons comparing 19 synaesthetes against their matched controls, computed using paired $T$-tests. We also computed the $95 \%$ CI of the odds ratio using mixed-effect generalized linear model. Results are very similar and shown in Supplemental Figures S4 and S5.

In order to fully exploit our data set, we performed two additional analyses.

A searchlight analysis was performed over the whole brain (Kriegeskorte et al., 2006). Whole brain analyses are in principle less powerful than ROI analyses because they constrain to distort each subject's anatomical space within one common space, so the average performance at any given voxel may in reality correspond to different anatomico-functional voxels in different subjects. Moreover, they re-introduce the methodological issues related to spatial smoothness (Stelzer, Lohmann, Mueller, Buschmann, \& Turner, 2014). This analysis was therefore exploratory. It allowed us to discover other clusters potentially involved in 
synaesthesia, which we could further analyse as post-hoc regions of interest to see if they displayed a consistent pattern of results across classifiers. The searchlight analysis used a 15 $\mathrm{mm}$ radius and the SVC algorithm. Performance maps were transformed to the common DARTEL space for voxel-wise group comparisons (resolution 3 by 3 by $3 \mathrm{~mm}$ ). We performed in SPM8 two-sample (groups of 20 subjects) and paired-sample $T$-tests $(\mathrm{N}=19)$ between synaesthetes and controls, as well as one-sample $T$-tests to compare the average performance in each group $(\mathrm{N}=20)$ against chance $(=0.25)$. For all comparisons, no individual voxel reached $P_{F W E}<0.05$. We used cluster-based statistics with the clusterforming threshold set to $P=0.001$ and $P_{F W E}<0.05$.

We also performed whole-brain univariate analyses on the groups of synaesthetes and controls to test for differences of magnitude of the BOLD responses to graphemes evoking synaesthetic colours. The design of the experiment was not optimized for these analyses since we did not have any control stimuli (those being not necessary for MVPA). The rationale was the same as for the whole brain searchlight analysis: if any difference was found between synaesthetes and controls, the revealed clusters could be defined as post-hoc regions of interest for our classifiers to test if those regions were involved in coding synaesthetic colours. A $9 \mathrm{~mm}$ FWHM spatial smoothing was applied to the subjects' EPI images before testing two contrasts: a $T$-contrast of all stimuli against the fixation point (we did not have graphemes that did not evoke any synaesthetic colour); an $F$-contrast of the four pairs of graphemes. Contrast maps were distorted within the study-specific template computed using DARTEL procedure as implemented in VBM8 (Dojat et al., 2018) and to the MNI space (resolution 1.5 by 1.5 by $1.5 \mathrm{~mm})$. For second-level analyses, we compared the contrast maps of synaesthetes $(\mathrm{N}=20)$ against controls $(\mathrm{N}=20)$ using $T$-tests (testing stronger signals either in synaesthetes or controls). We also performed paired $T$-tests on 19 synaesthetes against their matched control to account for possible differences due to the specific choices of graphemes in each 
synaesthete. For all comparisons, no individual voxel reached $P<0.05$, corrected for the family-wise error (FWE, based on the random field theory as implemented in SPM8). We used cluster-based statistics with the cluster-forming threshold set to $P=0.001$ (Eklund, Nichols, \& Knutsson, 2016) and $P_{F W E}<0.05$. As a final control analysis, we performed the same analyses for coloured stimuli.

\section{Results}

\section{Multivariate pattern analysis in regions of interest (defined at the individual level)}

Our main objective was to test if, in synaesthetes, some brain regions coded both real and synaesthetic colours. If neural representations are similar for real and synaesthetic colours, then classifiers trained on patterns of BOLD responses to distinguish real colours should predict which synaesthetic colours were experienced by synaesthetes when seeing achromatic letters and digits, while the performance of colour classifiers should be at chance level in control participants, for whom letters or digits do not evoke any synaesthetic colour. Figure 5 shows the result of the corresponding "colour to synaesthesia" ('C2S') classification in visual areas known to be involved in colour processing, retinotopic areas and the fusiform gyrus (ventral pathway). Performance was around the $25 \%$ chance level in every ROI for controls, as expected, but also for synaesthetes. The performance for each participant was within the 95\% limits of the binomial distribution (denoted by the thin green lines), except for six subjects where it was just slightly above (36\% in V1 for syn 12, 39\% in V2 for syn26, 39\% in V3 for syn02, 36\% and 39\% in V4 for respectively syn27 and syn26, 36\% in the subdivisions 1 and 2 of the right fusiform gyrus, FG12R, of the control subject of syn27, and $38 \%$ in the subdivisions 3 and 4 of the left fusiform gyrus, FG43L, of the control subject of syn11) and one subject where it was slightly below (14\% in FG12R for the control subject of syn01). 
Those high and low values are expected to occur by chance given multiple comparisons (20 subjects tested for each classification), all of them being well included within the $99.75 \%$ limits of the binomial distribution $([11,42] \%)$.

At the group level, mean performance could have been above chance even if still included within the $95 \%$ limits of the binomial distribution of individual performance (thin green lines). However, the $95 \%$ Confidence Interval (CI) of all groups reached or crossed the 0.25 chance baseline and remained below 30\%, meaning that the highest performances compatible with these data were quite low (See Methods: Statistical Analysis for a Bayesian interpretation). Moreover, there was no evidence of better classification in synaesthetes than in controls: whiskers across the zero blue line denote the $95 \%$ CI for paired comparisons of classifier performance of 19 synaesthetes against their matched control, the difference of performance being denoted by the grey crosses; all the $95 \%$ CIs of the group differences reached or crossed the zero line; the maximum higher performance of synaesthetes compatible with the data was $6 \%$ (in V2 and V3).

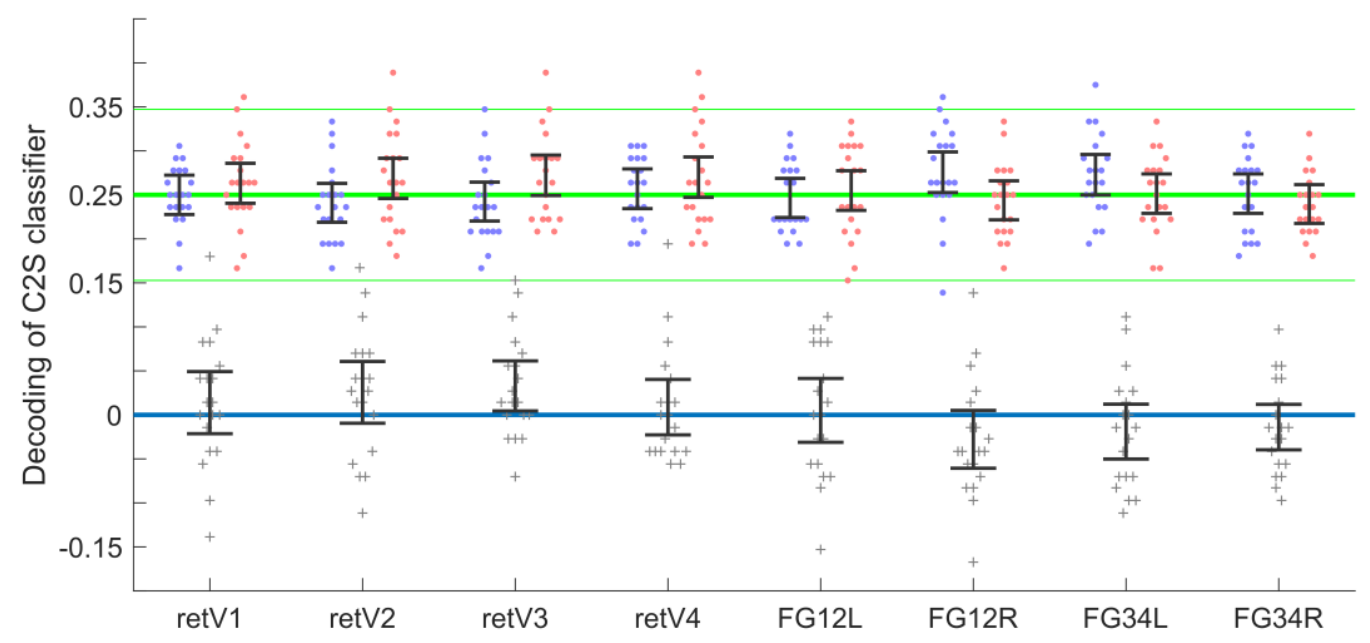

Figure 5. Performance of classifiers trained on real colours and tested on letters and digits evoking similar synaesthetic colours in synaesthetes (' $\mathrm{C} 2 \mathrm{~S}$ ' classification), in retinotopic areas and in the fusiform gyrus of synaesthetes (red points) and controls (blue points). Each classifier was trained and tested on beta weights computed on voxels in the native subject space with no spatial smoothing. The $\mathrm{y}$-axis represents both the performance of the ' $\mathrm{C} 2 \mathrm{~S}$ ' classifier (between 0 and 1 , chance level $=0.25$, 
thick green line; $95 \%$ limits of the binomial distribution of chance for each subject $[0.15,0.35]$, thin green lines) for individual subjects and their group average (with 95\% CI, computed using mixedeffect generalized linear models: see Methods, Statistical Analysis) and the difference of performance (grey crosses) between synaesthetes and their matched controls $(0=$ no difference between groups, blue line; whiskers denote 95\% CI, computed using paired T-tests: see Methods, Statistical Analysis). Each ROI regrouped several areas, for example the left and right parts of V1 for 'retV1' in order to provide a large number of voxels in each subject and ROI (at least $>60$ in each ROI of each subject, and > 100 voxels in most ROIs; see Methods: Data Analysis). ROIs have different number of voxels because no voxel selection was applied for this analysis. 'retV1' to 'retV4' were defined based on retinotopic mapping in each subject; other ROIs were defined as the intersection of the subject's grey matter mask and the mask of atlas-based anatomical ROIs (Anatomy Toolbox for SPM8) projected into the subject's space (see Figure 4). 'FG12L' = left (FG1 + FG2), etc. Please note that the scale of the $\mathrm{Y}$-axis, adjusted for better visibility, is different than in the next figures.

Figure 6 shows the performance of all classifiers (described in Figure 3) in all our ROIs, still without any voxel selection (ROIs have therefore different number of voxels across regions and subjects). 

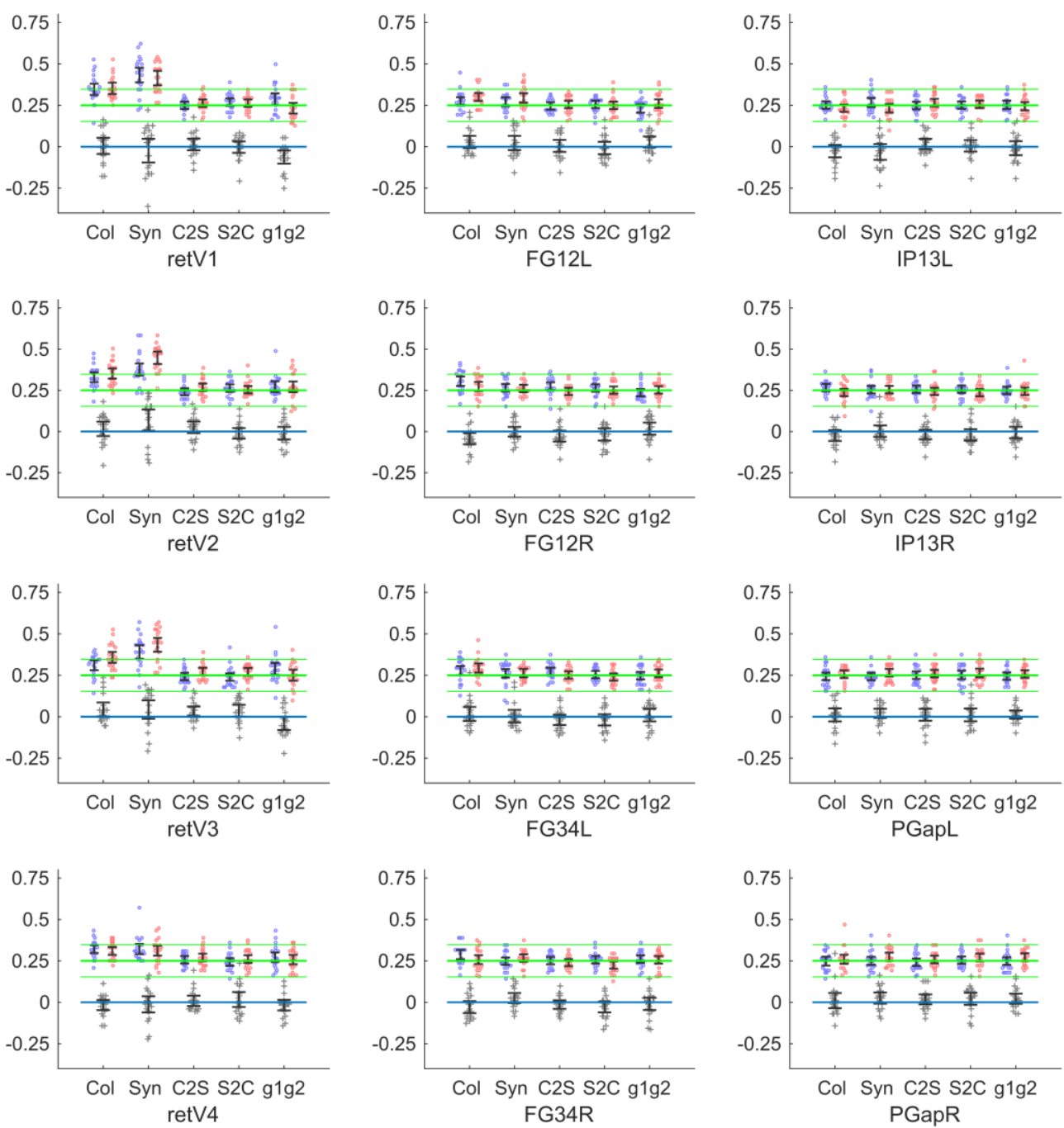

Figure 6. Decoding performance of all classifiers in retinotopic areas, the fusiform gyrus and parietal areas. Each panel displays the individual and average performances of five classifiers (see Figure 3), with the same conventions as in Figure 5: 'Col' = training and test on betas for real colours (rings); 'Syn' = training and test for synaesthetic colours (graphemes, letters or digits); 'C2S' = training on real colours (rings) test on synaesthetic colours (graphemes): these data were already shown in Figure 5 for retinotopic areas and the fusiform gyrus; ' $\mathrm{S} 2 \mathrm{C}$ ' = training on synaesthetic colours test on real colours; 'g1g2' training on letters test on digits or training on digits test on letters. As in Figure 5, the $\mathrm{y}$-axis represents both the performance of classifiers for individual subjects (blue $=$ controls, red $=$ synaesthetes) and the difference of performance (grey crosses) between synaesthetes and their matched controls. Whiskers around the group averages denote 95\% CI as computed for Figure 5. 'IP13L' = left (AIPS_IP1 + AIPS_IP2+ AIPS_IP3), 'PGapL' = left (IPL_PGa + IPL_PGp), etc. (see Figure 4). For 
the group differences, the CIs of the odds ratio computed by mixed-effect generalized linear models are shown in Supporting Information, Figure S4.

In each subplot, the first two beeswarms from the left show the performance of decoders in each subject for real colours ('Col' classification). As expected, the decoding of real colours was above chance $(0.25$, thick green line) in retinotopic areas as well as in the fusiform gyrus for both controls (blue points) and synaesthetes (red points). No difference was expected nor observed between groups. Note though that the whole $95 \%$ CI of the group difference was slightly above 0 in retinotopic V3 ('retV3'), and it was slightly below 0 in the subdivisions 1 and 2 of the right fusiform gyrus ('FG12R'). Differences are more visible when estimating the CI by a mixed-effect generalized linear models (Supporting Information, Figure S4). But without any independent evidence, these small differences could be due to random sampling. Indeed, all the $99.58 \%$ CIs included 0 (Bonferroni correction over 12 tests).

The next beeswarms are for the classification of pairs of graphemes. In synaesthetes only, classification could in principle be achieved based on the synaesthetic colours, since the synaesthetic colour was the main shared feature associated to each grapheme pair (in most cases one letter and one digit) had in common (so we called it the 'Syn' classification). For example, E and 7 were both associated to red by syn01 (see Figure 1). Performance was above chance level (25\%) in controls. This means that this performance could be achieved by classifiers based on either some spatial features shared by each grapheme pair or by optimizing decoding to only one of the graphemes. In order to test whether graphemes could be decoded on the basis of synaesthetic colours, we looked if classifiers performed better for synaesthetes than controls. This was the case in retinotopic V2 (95\% CI of the difference of performance, two-sample $T$-test: $[1.5,12.5] \%$; paired $T$-test: $[0.5,13.4] \%$; $95 \%$ CI of the odds ratio $[1.15,1.56]$; see Methods, statistical analysis) and to a lesser extent in retinotopic V3 (but note that performance was lower for synaesthetes in the subdivisions 1 to 3 of the left 
Intra-Parietal Sulcus, 'IP13L'; such a difference is most likely due to random sampling since none of the group performances in 'IP13L' was above chance). Only the difference in V2 survived Bonferroni correction over 12 tests, for the mixed-effect analysis $(P=0.0002)$.

The third group of beeswarms represents the data answering our main question: can synaesthetic colours be decoded based on real colours (' $\mathrm{C} 2 \mathrm{~S}$ ' classification)? These data were already displayed in Figure 5 (except for parietal ROIs), leading to a negative answer. In particular, performance was not significantly above chance in V2, as would have yet been expected if the higher performance in synaesthetes for the 'Syn' classification was really due to the coding of synaesthetic colours. We obtained similar null results when we tried to decode real colours based on graphemes (and possibly synaesthetic colours in synaesthetes: 'S2C' classification).

The last beeswarms on the right of each subplot show the performance for another classification that should have been possible only on the basis of synaesthetic colours: classifiers were trained on one set of graphemes (digits or letters) and tested on the other set of graphemes ('g1g2' classification). Again, performance was never above chance and never higher in synaesthetes than in controls, in particular in V2. We even observe lower scores for synaesthetes in V1 (where it even survived Bonferroni correction for the mixed-effect analysis: $P=0.0002$ ) and V3, where we had yet observed higher performance for 'Syn' decoding.

We performed again all these analyses using six larger ROIs (regrouping either the left or the right parts of V1 to V4, FG1 to FG2 and the areas of the inferior parietal lobule and the intraparietal sulcus) in which we selected the 100 voxels with the largest scores to $F$-tests to real colours, in order to feed the classifiers with the voxels most sensitive to real colours (Figure 7). The performance of the 'C2S', 'S2C' and 'g1g2' classifiers was never above 
chance in synaesthetes (nor in controls, as expected), and performance was never better in synaesthetes.
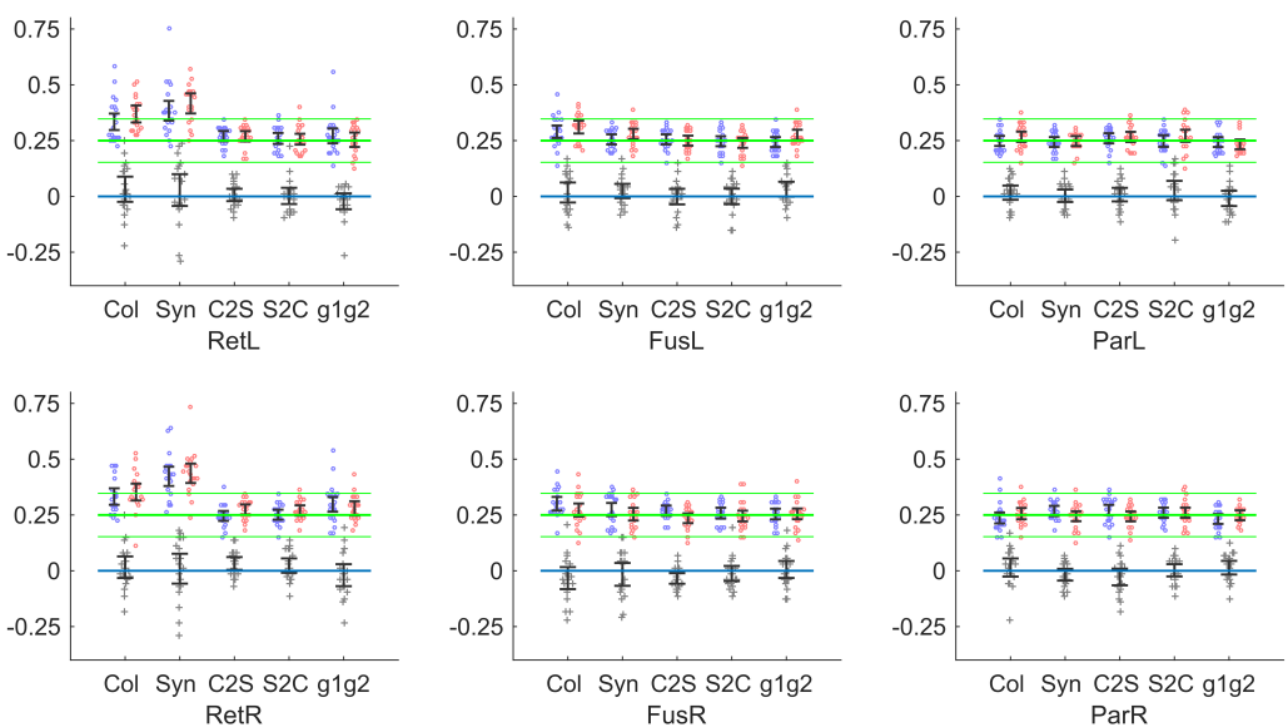

Figure 7. Decoding performance based on the same number of voxels $(=100)$ in each large ROI (retinotopic areas, fusiform gyrus and parietal regions) and subject. For the classification of real colours ('Col'), the selection of the best $F$-values to colours was different for each of the six leaveone-out classifications, based each time only on the five runs used for training the classifier, to insure independence of training and test. For the other selections, all colour runs were used to select the voxels with the highest $F$-scores. The high performance for the 'Syn' classification in retinotopic areas indicates that many voxels respond both to change of colour or luminance and the shape of graphemes, probably thanks to the small receptive fields of lower visual areas. Same conventions as in Figure 6. For the group differences, the CIs of the odds ratio computed by mixed-effect generalized linear models are shown in Supporting Information, Figure S5.

\section{Individual differences}

The phenomenological experience of synaesthetic colours may vary a lot across synaesthetes, which may compromise the visibility of effects at the group level. While this phenomenology has been so far problematic to capture with objective measures, we could estimate the strength of the synaesthetic associations in each subject, using variants of Stroop tasks. We reasoned that synaesthetes with stronger synaesthetic associations might have stronger modulations of 
the BOLD signal and thus larger decoding values. We first tested area V2, where we had observed on average higher performance in synaesthetes for the 'Syn' classification. We were wondering if this higher performance was really due to the coding of synaesthetic colours, because our other, more specific classifiers ('C2S', 'S2'C and 'g1g2'), had not revealed any difference. A correlation between synaesthetic strength and performance would constitute an independent validation of coding. Figure 8 shows the decoding performance in each subject as a function of the strength of synaesthetic associations, measured in Stroop-like psychophysics experiments (see Table 1 and Ruiz \& Hupé, 2015, for further explanations about the 'Photism Strength' index) for synaesthetes only (red crosses). Controls (blue circles) were attributed the value of their matched synaesthete.

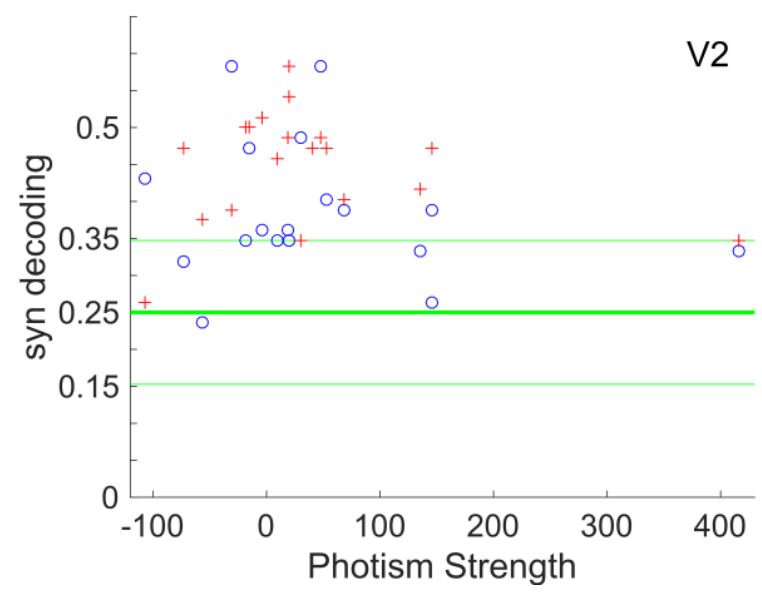

Figure 8. Decoding performance of the classifier trained and tested with synaesthetic colours (pairs of graphemes) in each subject in area V2 defined retinotopically (same data as 'Syn' in the second panel of the first column of Figure 6) as a function of the strength of synaesthetic associations ('Photism Strength'). This strength, measured for synaesthetes (red crosses), does not show any evidence of correlation with the performance of the 'Syn' decoder $(r=-0.11,95 \%$ CI [-0.54, 0.36]). Controls (blue circles) were attributed the value of their matched synaesthete $(r=-0.18,95 \%$ CI $[-0.58,0.30])$. Note that one value of Photism Strength was larger than the other ones. We carefully checked that this value was correct. However, given its possible influence on the correlation results, we complemented this analysis with two other analyses, by removing this value (for synaesthetes, $r=0.28,95 \%$ CI $[-0.21$, $0.66]$ ) and by performing non-parametric correlations (Spearman $r=-0.03, \mathrm{~N}=19, P=0.90$ ). We compared the results of these three statistical tests for all the other tested correlations. The statistical conclusions were always similar, except for one case described in Supporting Information, Figure S2. 
There was no correlation between both measures, neither for synaesthetes nor, as expected, for controls. We also observed that the difference of score between each synaesthete and her (or his) matched control did not increase with photism strength $(r=0.06,95 \%$ CI $[-0.42$, 0.52]; Spearman $r=0.01, \mathrm{~N}=18, P=0.95)$. Therefore, this analysis did not provide any independent argument in favour of the decoding of synaesthetic colours in V2. We computed similar correlation analyses in every ROI and for all classifiers and never found any correlation (all uncorrected $P>0.05$ ). We also computed both positive and negative (in case some regions would show a decrease of classifier performance for higher values of photism strength) correlations over the whole brain for the five classifiers, independently for synaesthetes and controls. We never found any significant cluster (cluster forming threshold, $P=0.001)$.

\section{Whole brain searchlight multivariate pattern analysis}

We complemented our ROI analysis with searchlight analyses over the whole brain (normalized to the MNI space), comparing the decoding performance in each group against chance as well as comparing groups for the five classifications. These exploratory analyses were performed to discover clusters potentially involved in synaesthesia outside of our ROIs. "Significant" clusters could be used as post-hoc regions of interest to see if they displayed a consistent pattern of results across classifiers (these results are displayed in Supporting Information, Table S1). We found no differences between controls and synaesthetes at our statistical threshold $\left(P_{F W E}<0.05\right.$ at the cluster level, cluster-forming threshold $\left.P=0.001\right)$ for classifiers trained and tested on colours (rings, 'Col' classifiers). For classifiers trained and tested on synaesthetic colours (graphemes, 'Syn' classifiers), we observed higher performance in synaesthetes in the parietal cortex (Table S1: on the right side with paired $T$-tests and on the left side with two-sample $T$-tests; bilateral difference could be observed for both contrasts when using a higher cluster-forming threshold). However, testing synaesthetes against chance 
revealed no cluster at our threshold around these coordinates of the parietal cortex (performance was above chance in both groups in the occipital cortex, as expected).

We found no difference between controls and synaesthetes at our statistical threshold for the critical test of shared coding of real and synaesthetic colours, when classifiers were trained on coloured rings and tested on graphemes (' $\mathrm{C} 2 \mathrm{~S}$ ' classifiers). Testing synaesthetes against chance also revealed no cluster. The reverse classification (learning on graphemes, 'S2C'), however, revealed two clusters with higher performance in synaesthetes for independent $T$ tests, in the right occipito-temporal cortex and in the left putamen. Only the first cluster was confirmed by paired-comparisons. When testing performance against chance two clusters emerged for synaesthetes (none for controls), one again in the same part of the right occipitotemporal cortex, and the other in the left parietal cortex, abutting the parietal cluster obtained previously for the higher performance in synaesthetes for the 'Syn' classification (we shall come back to this concordance in the following post-hoc analysis).

Finally, for classifiers trained on either letters or digits (and tested respectively on either digits or letters), a critical test of the coding of synaesthetic colours, higher performance was observed, but in controls, in the left inferior frontal gyrus, for both paired and independent $T$ tests. However, no cluster emerged anywhere in the brain in controls (nor in synaesthetes) when testing performance against chance, so this cluster should be considered as a false positive.

Post-hoc analysis. We further explored the performance of classifiers in the two clusters identified by the 'Syn' classifier and the five clusters identified by the ' $\mathrm{S} 2 \mathrm{C}$ ' classifier, corresponding in fact to two parietal regions (left and right), one right occipito-temporal region and one cluster in the left putamen. In each cluster, we computed the average across voxels of the searchlight scores, in order to compare the performances of our five classifiers for synaesthetes and controls in these seven clusters defined post-hoc, with two-sample and 
paired-sample $T$-tests. We also compared the decoding performance in each group against chance. Statistically "significant" differences were obtained only for the contrasts used to define the clusters (Table S1). Only one additional comparison was "significant" $(P=0.012$, not corrected for multiple comparisons) in the left parietal cluster at XYZ $=\left[\begin{array}{ll}-33 & -2850\end{array}\right.$, which had been obtained when testing synaesthetes against chance for the ' $\mathrm{S} 2 \mathrm{C}$ ' classification (training classifiers on graphemes and testing them on colours: Figure 9): scores were also higher in synaesthetes than controls for decoding graphemes ('Syn' classification, 95\% CI [1, 9]\%, paired comparisons) and higher than chance (95\% CI [26, 31]\%), but the performance was not correlated with the strength of synaesthetic associations $(P=0.51)$.
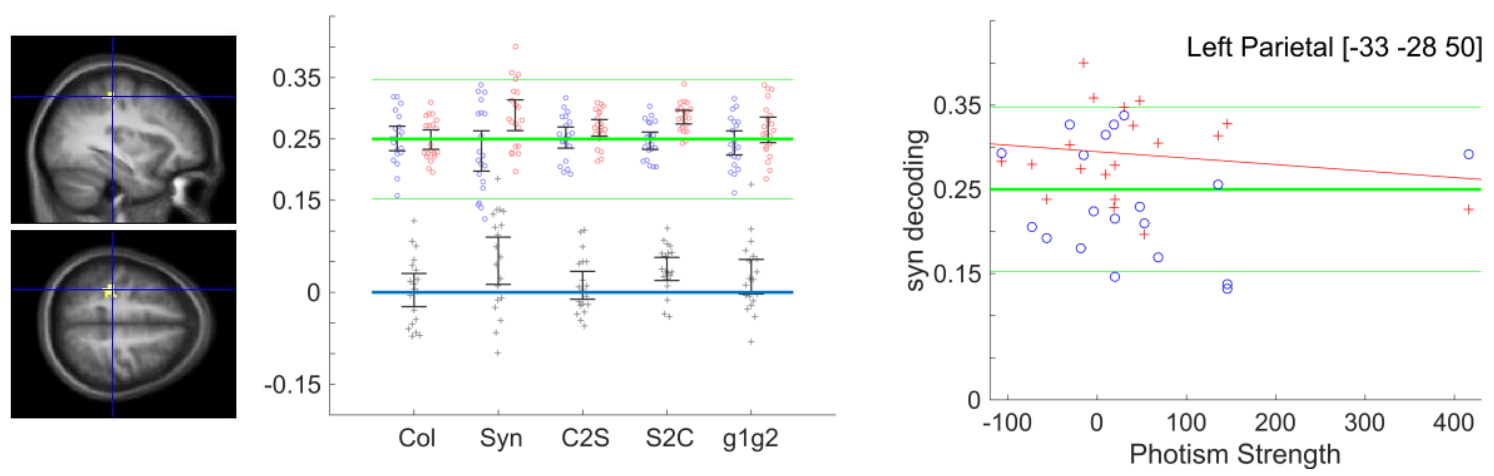

Figure 9. Left: Parietal cluster identified based on whole brain searchlight analysis for 'S2C' decoding, Synaesthetes $>$ chance (27-voxel cluster at XYZ $=\left[\begin{array}{l}-33 \\ -28\end{array}\right.$ 50], one-sample $T$-test). Middle: performance of classifiers in this cluster (same conventions as in Figure 6). The decoding performance for synaesthetes was logically above chance for the 'S2C' classification, since the cluster was defined based on this contrast. For the independent classifier 'Syn', the performance for synaesthetes was also above chance and above that of controls. Right: Absence of correlation between the strength of synaesthetic associations and 'Syn' decoding (Spearman $r=0.02, \mathrm{~N}=19, P=0.95$; for 'S2C' decoding, not shown: Spearman $r=-0.15, \mathrm{~N}=19, P=0.53)$.

\section{Whole brain univariate analyses (normalized anatomical space)}

Similarly to the searchlight analyses, these whole-brain univariate exploratory analyses were performed to discover clusters potentially involved in synaesthesia outside of our ROIs. 
We compared the maps of $T$-contrasts obtained for graphemes in synaesthetes and controls: $T$ contrasts reveal voxels which are most activated by any grapheme; the additional synaesthetic colour associated to graphemes in synaesthetes could lead to stronger BOLD signal. The whole-brain analysis revealed no difference between controls and synaesthetes at our statistical threshold $\left(P_{F W E}<0.05\right.$ at the cluster level, cluster-forming threshold $\left.P=0.001\right)$ when performing two-sample $T$-tests between controls and synaesthetes. However, the pairedsample $T$-tests revealed stronger BOLD signal in a small cluster in synaesthetes, close to the left precentral gyrus. We treated this cluster as a candidate region for the coding of synaesthetic colours (Supporting Information, Table S1).

We compared the maps of $F$-contrasts obtained for graphemes in synaesthetes and controls: $F$-contrasts reveal voxels which are most modulated by graphemes; the additional synaesthetic colour associated to graphemes in synaesthetes could lead to larger differentiations of BOLD signals. The whole-brain analysis did not reveal any stronger modulation in synaesthetes than controls (neither for two-sample nor paired-sample $T$-tests). Surprisingly, we observed stronger modulation in controls in two clusters (paired comparisons), in the right occipito-parietal cortex (Supporting Information, Figure S1) and in the left insula. The two-sample $T$-tests revealed only the occipito-parietal cluster. We did not have any explanation for these differences, which might be false-positives (Eklund et al., 2016). We note that the analysis by (Rouw \& Scholte, 2010) revealed a cluster (which they called IPS, cluster extent $=3280 \mathrm{~mm} 3)$ at equivalent peak coordinates on the left side $([-30$ 72 28]), obtained with the contrast synaesthetes > controls for (synaesthetic graphemes) > (non-synaesthetic graphemes). In our case, the weaker modulation by graphemes in synaesthetes would rather argue against the hypothesis of a functional role of this region in synaesthesia. We included these two regions in our post-hoc MVPA analyses for further exploration. 
We also compared synaesthetes and controls for the maps of $T$ - and $F$-contrasts for the responses to real colours (rings), in case the presence of synaesthesia would lead to a different processing of real colours. We observed stronger BOLD signal ( $T$-contrast) in synaesthetes only, in three clusters for paired comparisons (in the left posterior and anterior insula - see Supporting Information, Figure S2 - and in the left parahippocampal region) and two other clusters for two-sample $T$-tests (in the right middle temporal gyrus and in the right superior, medial, frontal gyrus - see Supporting Information, Figure S3). The lack of consistency between paired and two-sample $T$-tests could again suggest false-positives, but we nonetheless included these five clusters in our post-hoc MVPA analyses, in case those stronger activations be related to the implicit activation of graphemes by the colours associated to them ("bi-directional" synaesthesia: Gebuis, Nijboer, \& Van der Smagt, 2009). $F$-contrasts to colours revealed only one cluster of stronger modulation in controls in the frontal region, but in the middle of white matter and thus clearly a false positive.

We compared the performances of our classifiers in synaesthetes and controls in those eight clusters defined post-hoc, with two-sample and paired-sample $T$-tests. Only three comparisons came out "significant" at $p<0.05$, but without correction for multiple comparisons (Table S1). Supporting Information, Figures S1 to S3 detail the results obtained in these three posthoc clusters.

\section{Discussion}

Our goal with this study was to provide univocal evidence of the activation of the 'colour network' by imaginary colours as experienced by grapheme colour synaesthetes.

Studies based on univariate analyses to search the neural correlates of synaesthetic colours face two major problems. First, BOLD responses to stimuli leading to the experience of 
synaesthetic colours need to be compared to a control response (subtraction method: see the Figure 1 by Hupé \& Dojat, 2015). Such a control response may be obtained by testing the same subjects with similar stimuli that do not generate a synaesthetic experience (pseudographemes or graphemes that, by chance, do not generate such an experience in the tested synaesthetes). The problem is, it is impossible to know whether the additional activations, if observed, are specific to the synaesthetic experience of colours. For example, letters and numbers can also be named, unlike pseudo-graphemes. A control response may also be obtained by testing non-synaesthetes with the same stimuli. But, again, it is impossible to know whether the additional activations, if observed, are specific to the synaesthetic experience of colours. For example, synaesthetes often enjoy visualizing the synaesthetic colours of graphemes: attentional and emotional components may therefore bias the comparison. Second, averaging the results across subjects require to transform the individual data within a common reference space, with the possible loss of fine-grained spatial information. The first problem may generate false positive results, the second false negative results.

Thus, because univariate analysis led to inconsistent results (Hupé \& Dojat, 2015), we used in this study Multivariate Pattern Analysis (MVPA) on 20 synaesthetes and 20 control participants, to explore whether the neural processing of real colours and synaesthetic colours shared patterns of activations. To our knowledge, it was the first time that MVPA was proposed in this context. By using MVPA, we could in principle overcome problems associated with univariate analysis because we test the performance, in each individual, related to the coding of certain attributes, not a level of activation in need of further interpretation. Our design was optimized to test if classifiers, trained to distinguish patterns of responses to four different real colours in groups of voxels from different regions of the brain, could classify above chance the responses of those voxels to achromatic graphemes leading to 
the synaesthetic experience of the exact same colours. The logic was that only synaesthetes tested with their exact, idiosyncratic, synaesthetic code could produce above-chance performance. But the classifier performance in our group of 20 synaesthetes remained very close to chance (all $P>0.05$, uncorrected) in all our selections of voxels (retinotopic areas defined at the individual level as well as the fusiform gyrus and parietal regions of interest defined based on a probabilistic atlas), and whatever the extent of the chosen areas or the selection method of the voxels (Figure 5 and ' $\mathrm{C} 2 \mathrm{~S}$ ' classification of Figure 6 and 7, shown by red points for synaesthetes). A statistical comparison revealed that the classifier performance in synaesthetes was also no better on average than that obtained in control subjects.

The absence of statistically significant effect cannot lead to conclude to the absence of effects. The null result could be due to a lack of power, if, for example, a good performance of classifiers was reached for only a few synaesthetes. However, the results were not ambiguous when inspecting the distributions of classifier performances in individual subjects: the scores for most synaesthetes were distributed around the chance level (binomial probability: almost all the points are included within the green dotted lines representing the $95 \%$ limits of the binomial distribution). The correlation analyses with a measure of individual differences (the strength of the synaesthetic associations) further confirmed the homogenous performance of classifier around chance for synaesthetes. There is therefore no evidence at all in the present data of shared coding of real and synaesthetic colours.

We further analysed our data set in different ways, in order to be able to detect some signs of coding of synaesthetic colours by neural networks not involved in the coding of real colours. The 'g1g2' classification, which could have been achieved only on the basis of shared synaesthetic colours across letters and digits, remained at the chance level in synaesthetes. The 'syn' classification, expected to reach a higher performance in synaesthetes, was similar in controls. We also explored the performance of classifiers beyond our regions of interest, 
across the whole (normalized) brain (searchlight analysis) without obtaining significant results.

We also explored the whole brain using mass univariate tests, knowing that whole brain analyses face the ill-posed problem of correction of multiple comparisons of partly correlated tests, problem not fully solved by the Random Field Theory (Eklund et al., 2016). Moreover, since we performed in total at least nine whole brain searchlight analyses and four whole brain univariate comparisons ( $T$ - and $F$-contrasts for responses to graphemes and colours, see Table S1), we could have set a family-wise error level at $0.05 / 13$. We preferred to keep a noncorrected level for easier comparisons with other studies. The whole brain analyses were used only for exploration, and for every detected cluster we searched for additional evidence (differential response for other comparisons, or correlation with individual differences). Since we did not find any additional evidence, we conclude that these clusters may be false positives. We however mention them (see Table S1) in case additional evidence be found in other studies.

If considering synaesthetic colours simply as a form of mental imagery, as proposed in the Introduction, we were expecting above-chance decoding performance as observed for other tasks involving mental imagery. Those other tasks, however, typically involved different categories of objects, like food, tools, faces and buildings (Reddy et al., 2010) or objects, scenes, body parts and faces (Cichy, Heinzle, \& Haynes, 2012), which evoked stronger BOLD signal in specific areas (like the Fusiform Face Area). Other studies involved retinotopic properties (Thirion et al., 2006) where, again, differences of BOLD signal can be easily observed. Here we were trying to decode mental images within only a single category, colour. Our negative results suggest that synaesthetic experiences do not evoke strong BOLD responses, as already suggested by the inconsistency of the published results based on univariate models (Hupé \& Dojat, 2015). 
Below we consider alternative explanations (e.g., methodological limitations) to the absence in our data of shared patterns of activations for real and synaesthetic colours as well as of neural traces of the processing of synaesthetic colours. These limitations suggest alternative methodological choices for further experiments.

\section{Colour classifiers used luminance differences rather than colour differences.}

The colours of the stimuli were based on the choices made by synaesthetes, corresponding to their synaesthetic colours. Those were of course not isoluminant, especially when comparing blue and yellow. Then, classifiers may have learnt luminance rather than hue differences, resulting in "luminance" decoders rather than "colour" decoders as we expected, and an impossibility of testing our colour hypothesis. However, such luminance decoders should have been able to pick up the luminance difference of synaesthetic colours in case of shared coding between real and synaesthetic perception, and the conclusions of our study would therefore be similar. In any case, it is unlikely that luminance signals were stronger than hue signals everywhere in the visual cortex. Luminance coding concerns mostly low-level retinotopic areas while high level areas are sensitive to unique hues (Stoughton \& Conway 2008). Note that hue differences are often perceptually more salient than luminance differences (see Figure 1); therefore, corresponding signals must exist in the brain, which our classifiers should have been able to pick up. In the Methods section, we evaluated the information content of our stimuli as measured in the $\mathrm{L}^{*} \mathrm{a} * \mathrm{~b}$ colour space, supposed to be approximately homogenous for colour distances (see Figure 2). We concluded that, within that space, colour classifiers would use hue rather than luminance cues. Nonetheless, since we do not know what colour space if any is the most appropriate to identify the neural correlates of colour processing, it could be interesting to redo our experiment by choosing only approximately isoluminant synaesthetic colours. Inspection of our Figure 2 shows that this 
would have been possible at the expense of restricting our study to two colour categories, like red and green or yellow and green in most subjects, red and blue in a few others.

\section{Colour imagery could not be decoded.}

Our study used a protocol very similar to that used by (Bannert \& Bartels, 2013), who could decode the typical colour from eight objects, presented as greyscale photos, with classifiers trained on concentric colour circles designed after (Brouwer \& Heeger, 2009), like in our study. The prototypical colour of the objects was red, green, blue or yellow (like a banana and a tennis ball). Across 18 subjects, decoding accuracy was "significantly" above chance in V1, but reached only $32 \%$ on average, which is hardly above the $95 \%$ CI $([24,30] \%)$ of the performance observed for our similar classifier (' $\mathrm{C} 2 \mathrm{~S}$ ') in the areas V1 to V4 of synaesthetes. Their experimental procedures, slightly different from ours, may have better optimized the signal to noise ratio and allowed this higher performance (see below). Alternatively, since the colour-diagnostic objects were presented before the coloured concentric rings, subjects may have imagined, when viewing the rings, the very objects that were presented before. Subjects had to do a motion discrimination task to divert their attention (similarly to our one-back task), but such a task (like ours) was not very demanding (though note that Bannert and Bartels argue that their results are due to automatically occurring processes during object vision rather than active imagery). A similar argument holds even more in our experiment: synaesthetes were very likely to recognize the colour matching exactly their synaesthetic colour of letters and digits, and they might well have imagined the letter or digit when looking at the coloured stimuli. In both cases, decoding would be based on the complex shape of stimuli rather than their colour. In the case of Bannert and Bartels, objects were similar to those used in other successful visual-to-imagery decoding and involved several categories of objects as well as different retinotopic properties (the objects had different orientations but were rotating; however the banana or the coke can, for example, had about 12 deg extent, 
apparently much more than the Nivea tin or the blue traffic sign), while in our case objects all belonged to the grapheme category, and all spanned the same visual extent. It is therefore possible that in the study by Bannert and Bartels the slightly above chance decoding performance was due to residual category and retinotopic properties, not to colour. With such an interpretation, decoding of imaginary colours would have failed in both their and our study.

Questionable choice in the paradigm used: duration of event presentation.

Our close to chance performance could be linked to our choice of a fast event related paradigm, each stimulus being presented each time for only $1 \mathrm{~s}$, with an ISI $=1 \mathrm{~s}+/-333 \mathrm{~ms}$. Bannert and Bartels presented images for $2 \mathrm{~s}$ with a $1 \mathrm{~s}$ ISI, each repeated four times in a row (miniblocks). One may wonder whether our presentation time was sufficient to trigger synaesthetic associations. However, psychophysical tasks show that the naming of the synaesthetic colours of graphemes takes on average much less than $1 \mathrm{~s}$ (Table 1). Because of our one-back attentional task, though, we cannot be sure that the synaesthetic associations were always conscious. However, synaesthetes did not report any specific difficulty with their synaesthetic experience when viewing, inside the scanner, the proposed paradigm. We designed such a protocol because we did not want synaesthetes to pay too much attention to their synaesthetic colours, then possibly triggering complex attentional and emotional processes. Those components are part of the synaesthetic experience, but they do not tell us anything about the phenomenological experience of colours, our main goal being to try to isolate the possible neural commonalities of the real and synaesthetic experience. The quasiabsence of observed differences of overall activation and modulation between synaesthetes and controls for graphemes indicates that we were successful in synaesthetes having a similar experience to controls for graphemes, in terms of attentional and emotional content. With different conditions, favouring synaesthetic colours to be experienced intensely, we would 
expect the overall pattern of brain activity to be different, but those differences would be poorly informative. However, those differences may not play any role in the specific MVPA decoding of synaesthetic colours based on real colours (' $\mathrm{C} 2 \mathrm{~S}$ ' classification), except for the possible sub-vocalization of colour names. A protocol with longer presentation time and a task related to synaesthetic colours could therefore be tried, if sub-vocalization of colour names is prevented by a different protocol and task when presenting real colour stimuli.

Questionable choice in the paradigm used: type of stimuli.

A critical aspect of our fast-event paradigm is related to the slow dynamics of the hemodynamic signal and the signal to noise we could obtain. Here, the critical benchmark was the possibility to decode real colours, since the protocol was identical for synaesthetic and real colours. We were successful in decoding colours above chance in the visual cortex, between 31 and $36 \%$ in V1 to V4, chance being 25\%. Using 12 s miniblocks, Bannert and Bartels obtained an average performance for colours between $35 \%$ and $40 \%$ in V1 to V4. Differences other than the timing of the stimuli may explain this only slightly higher performance: their total presentation time of coloured stimuli was about $42 \mathrm{~min}$ (20 min in our study; for example, Brouwer \& Heeger, 2009, obtained even higher performances with experienced participants tested for much longer durations); their stimuli were much larger (7.19 deg vs. 2 deg radius) and isoluminant (we do not know whether luminance information in our case helped or hindered decoding). Because they were constrained by the idiosyncratic synaesthetic associations, our stimuli were also not well distributed within the colour space (see Figure 2). Colour differences between categories (R, G, B and Y) and similarity between colours for pairs (letter-digit) were different between subjects and not always optimal to reach maximal performance by classifiers. Probably, some pairs of supposedly similar colours confused classifiers, as well as short distances between some categories. Choosing only three colours would have allowed us to avoid confusions and get more exemplars for each colour 
(with fewer categories to decode, though, confounding factors are more likely). More repetitions would have increased the signal to noise ratio, however we wanted to record signals for real and synaesthetic colours within the same scanning session to avoid any spatial smoothing of the voxels (which is often necessary when aligning images obtained in different sessions). Preliminary experiments had showed us indeed that combining the signals from different sessions did not improve performance (Ruiz et al., 2012). Our total session time was about 1 hour, which is about the limit one may ask naïve subjects to lie in a scanner without moving while maintaining fixation and attention.

\section{Lack of statistical power to detect small differences.}

Given our moderate performance for colour classification, our absence of above-chance performance for the decoding of synaesthetic colours might be due to a lack of power, since performance across real and imaginary images is typically lower than for real images (Reddy et al., 2010; Bannert \& Bartels, 2013). However, we did have enough power to assess that performance for synaesthetic colours, if above chance, was less than half than for real colours: in the large ROI comprising the left retinotopic areas (retL), the average performance for decoding colour was $37 \%, 12 \%$ above chance. If performance was half that size for decoding synaesthetic colours based on real colours ('C2S'), performance should have been $31 \%$. It was only $27 \%$, with the $95 \%$ CI $[24.7,29.2] \%$. Bayesian analysis (see Methods: statistical analysis) indicates that there is $99.9 \%$ chance that the true performance of the ' $\mathrm{C} 2 \mathrm{~S}$ ' classifier in the left retinotopic areas is less than $31 \%$. In all our tested ROIs, the upper limit of the $95 \%$ CI of the 'C2S' classifier was always less than 30\%. Also, such a reasoning holds for the average performance, but some subjects did reach performance for colour decoding well above $50 \%$. Yet, the distribution of individual scores were all very similar for controls and synaesthetes (see Figs. 5 to 7). For example, if we select the 11 synaesthetes whose performance was above $35 \%$ (that is, above the chance level at the individual level) for 
decoding real colours in the left retinotopic areas ( retL; mean performance $=43 \%$ ), their ' $\mathrm{C} 2 \mathrm{~S}$ ' performance was no better than that of the whole group (mean $=27.5 \%, 95 \%$ CI [24.7, 30.3]\%; Bayesian analysis: $98.9 \%$ chance that the true performance is less than $31 \%$ ). In fact, there was no evidence for any correlation between the performances of colour classifiers and of synaesthetic classifiers based on real colours ('C2S'), in any of our ROIs (all uncorrected $P$ $>0.14,-0.36<R<0.35)$. There was some correlation between the performances of colour ('Col') and synaesthetic ('Syn') classifiers in retinotopic areas (especially V1), but it was similar in synaesthetes and controls (the differences between synaesthetes and controls for the 'Syn' classifier were in fact even weaker when including the 'Col' performance as a covariate).

For the statistical analysis, we adopted the "new statistical approach" proposed by (Cumming, 2012) and focussed on confidence intervals of effect sizes instead of the less informative thresholded $P$-value maps (Hupé, 2015). In order to facilitate the comparison of our study with previous studies, we indicated when the comparisons could be considered as "significant" (a 95\% CI not crossing the chance level corresponds to $P<0.05$ ) when correcting the risk level for multiple comparisons. Note however that correction for multiple comparisons corresponds to an ill-posed problem, because there is no unique and objective way to define the family of tests (Hupé, 2015). Such a problem is pretty obvious in our case, where the number of considered ROIs depends on our choice of regrouping or not ROIs, and by how much. We applied a Bonferroni correction over twelve ROIs, but we could have considered the family across the five types of classifiers (so at least 60 tests). However, by focusing on the extent of the CIs, the conclusions do not change much for different levels of CI (the extent of a $99.58 \%$ CIs is just a bit larger than for a 95\% CI): for all the cases that may suggest differences between groups, the true differences compatible with our observations may be either close to absent (difference close to or including 0 , or odds ratio close to or 
including 1 ) or at most up to about $15 \%$ (or odds ratio $=1.5$ ), a value that one may consider meaningful. As in most studies currently published in cognitive neuroscience dealing with small effects, the width of our confidence intervals is too wide to reach any definitive conclusion on the sole basis of one test (lack of power). Our choice of CI presentation, however, brings useful information allowing cumulative science (Yarkoni, Poldrack, Van Essen, \& Wager, 2010) and shows that if any real difference exists, it is probably not very large because corresponding to less than a $15 \%$ difference of performance.

\section{Conclusion}

Using MVPA, we could not identify the neural correlates of the synaesthetic experience of colours and we did not find any evidence of common neural coding of real and synaesthetic colours. By stressing this lack of evidence, we do not conclude that such a neural coding does not exist. We bring to light what is required to have any chance to reveal the neural bases of the synaesthetic experience using MRI, like more data by subject, higher signal to noise ratio and spatial resolution (e.g., 7 Tesla scanner: Turner, 2016), maybe larger cohorts or a different protocol addressing the issue of luminance differences or with a slower presentation rate. 


\section{Acknowledgements}

Research funded by Agence Nationale de la Recherche ANR-11-BSH2-010. Mathieu J. Ruiz was supported by a Ph.D. allowance from the Université Grenoble Alpes. Grenoble MRI facility IRMaGe was partly funded by the French program Investissement d'avenir run by the Agence Nationale de la Recherche: grant Infrastructure d'avenir en Biologie Santé ANR-11INBS-0006.

\section{Conflict of Interest Statement}

The authors declare no competing interests, financial or non-financial.

\section{Author Contributions}

M.J.R., M.D, and J.M.H. designed research; M.J.R. and M.D. performed research; M.J.R., M.D, and J.M.H. analysed data; J.M.H. wrote the paper; M.J.R., M.D, and J.M.H. revised the paper.

\section{Data Accessibility Statement}

The datasets generated and analysed during the current study are freely available on request (https://shanoir.irisa.fr/Shanoir/login.seam), contact M. Dojat. The data are not publicly available due to privacy restrictions, stated in the document approved by the Institutional Review Board. 


\section{Abbreviations}

CI: Confidence Interval

fMRI: functional Magnetic Resonance Imaging

FWE: Family-Wise Error

FWHM: Full Width at Half Maximum

GLM: General Linear Model

MVPA: MultiVariate (and MultiVoxel) Pattern Analysis

ROI: Region Of Interest

Meaning of the code names of the classifiers (see Figures 3 and 6 )

Col: training and test on betas for real colours (rings)

Syn: training and test for synaesthetic colours (graphemes: letters or digits)

C2S: training on real colours (rings) test on synaesthetic colours (graphemes)

S2C: training on synaesthetic colours test on real colours

g1g2: training on letters test on digits or training on digits test on letters. 


\section{References}

Bannert, M.M. \& Bartels, A. (2013). Decoding the yellow of a gray banana. Curr. Biol., 23(22), 2268-2272. doi: 10.1016/j.cub.2013.09.016

Bates, D., Mächler, M., Bolker, B. \& Walker, S. (2015). Fitting linear mixed-effects models using lme4. J. Stat. Soft., 67(1), 48. doi: 10.18637/jss.v067.i01

Bordier, C., Hupé, J.M. \& Dojat, M. (2015). Quantitative evaluation of fMRI retinotopic maps, from V1 to V4, for cognitive experiments. Front. Hum. Neurosci., 9, 277. doi: 10.3389/fnhum.2015.00277

Brouwer, G.J. \& Heeger, D.J. (2009). Decoding and reconstructing color from responses in human visual cortex. J. Neurosci., 29(44), 13992-14003. doi:

10.1523/JNEUROSCI.3577-09.2009

Caspers, J., Zilles, K., Eickhoff, S.B., Schleicher, A., Mohlberg, H. \& Amunts, K. (2013). Cytoarchitectonical analysis and probabilistic mapping of two extrastriate areas of the human posterior fusiform gyrus. Brain Struct. Funct., 218(2), 511-526. doi: $10.1007 / \mathrm{s} 00429-012-0411-8$

Caspers, S., Eickhoff, S.B., Geyer, S., Scheperjans, F., Mohlberg, H., Zilles, K. \& Amunts, K. (2008). The human inferior parietal lobule in stereotaxic space. Brain Struct. Funct., 212(6), 481-495. doi: 10.1007/s00429-008-0195-z

Caspers, S., Geyer, S., Schleicher, A., Mohlberg, H., Amunts, K. \& Zilles, K. (2006). The human inferior parietal cortex: cytoarchitectonic parcellation and interindividual variability. NeuroImage, 33(2), 430-448. doi: 10.1016/j.neuroimage.2006.06.054 
Chiou, R. \& Rich, A.N. (2014). The role of conceptual knowledge in understanding synaesthesia: Evaluating contemporary findings from a 'hub-and-spoke' perspective. Front. Psychol., 5. doi: 10.3389/fpsyg.2014.00105

Chiou, R., Rich, A.N., Rogers, S. \& Pearson, J. (2018) Exploring the functional nature of synaesthetic colour: Dissociations from colour perception and imagery. Cognition, 177, 107-121. doi: 10.1016/j.cognition.2018.03.022

Choi, H.J., Zilles, K., Mohlberg, H., Schleicher, A., Fink, G.R., Armstrong, E. \& Amunts, K. (2006). Cytoarchitectonic identification and probabilistic mapping of two distinct areas within the anterior ventral bank of the human intraparietal sulcus. J. Comp. Neurol., 495(1), 53-69. doi: 10.1002/cne.20849

Chun, C.A. \& Hupé, J.M. (2013). Mirror-touch and ticker tape experiences in synesthesia. Front. Psychol., 4, 776. doi: 10.3389/fpsyg.2013.00776

Chun, C.A. \& Hupé, J.M. (2016). Are synesthetes exceptional beyond their synesthetic associations? A systematic comparison of creativity, personality, cognition, and mental imagery in synesthetes and controls. Brit. J. Psychol., 107, 397-418. doi: 10.1111/bjop. 12146

Cichy, R.M., Heinzle, J. \& Haynes, J.D. (2012). Imagery and perception share cortical representations of content and location. Cereb. Cortex, 22(2), 372-380. doi: 10.1093/cercor/bhr106

Cox, D.D. \& Savoy, R.L. (2003). Functional magnetic resonance imaging (fMRI) "brain reading": detecting and classifying distributed patterns of fMRI activity in human visual cortex. NeuroImage, 19(2 Pt 1), 261-270.

Cumming, G. (2012). Understanding The New Statistics: Effect Sizes, Confidence Intervals, and Meta-Analysis. New York: Routledge. 
Dehaene, S. \& Cohen, L. (2011). The unique role of the visual word form area in reading. Trends Cogn. Sci., 15(6), 254-262. doi: 10.1016/j.tics.2011.04.003

de Valpine, P., Turek, D., Paciorek, C. J., Anderson-Bergman, C., Lang, D. T. \& Bodik, R. (2017). Programming with models: writing statistical algorithms for general model structures with NIMBLE. Journal of Computational and Graphical Statistics, 26(2), 403-413. doi: 10.1080/10618600.2016.1172487

Dojat, M., Pizzagalli, F. \& Hupé, J.M. (2018). Magnetic resonance imaging does not reveal structural alterations in the brain of grapheme-color synesthetes. PLoS ONE, 13(4), e0194422. doi: 10.1371/journal.pone.0194422

Eagleman, D.M., Kagan, A.D., Nelson, S.S., Sagaram, D. \& Sarma, A.K. (2007). A standardized test battery for the study of synesthesia. J. Neurosci. Methods, 159(1), 139-145. doi: 10.1016/j.jneumeth.2006.07.012

Edquist, J., Rich, A.N., Brinkman, C. \& Mattingley, J.B. (2006). Do synaesthetic colours act as unique features in visual search? Cortex, 42(2), 222-231.

Eickhoff, S.B., Paus, T., Caspers, S., Grosbras, M.H., Evans, A.C., Zilles, K. \& Amunts, K. (2007). Assignment of functional activations to probabilistic cytoarchitectonic areas revisited. NeuroImage, 36(3), 511-521. doi: 10.1016/j.neuroimage.2007.03.060

Eklund, A., Nichols, T.E. \& Knutsson, H. (2016). Cluster failure: Why fMRI inferences for spatial extent have inflated false-positive rates. Proc. Natl. Acad. Sci. USA, 113(28), 7900-7905. doi: 10.1073/pnas.1602413113

Flournoy, T. (1893). Des Phénomènes de Synopsie (Audition Colorée): Photismes, Schèmes Visuels, Personnifications. Paris: Alcan.

Formisano, E. \& Kriegeskorte, N. (2012). Seeing patterns through the hemodynamic veil--the future of pattern-information fMRI. NeuroImage, 62(2), 1249-1256. doi:

10.1016/j.neuroimage.2012.02.078 
Friston, K.J., Zarahn, E., Josephs, O., Henson, R.N. \& Dale, A.M. (1999) Stochastic designs in event-related fMRI. Neuroimage, 10, 607-619. doi: 10.1006/nimg.1999.0498

Galton, F. (1880). Statistics of mental imagery. Mind, 5, 301-318.

Gebuis, T., Nijboer, T.C. \& Van der Smagt, M.J. (2009). Multiple dimensions in bidirectional synesthesia. Eur. J. Neurosci., 29(8), 1703-1710. doi: 10.1111/j.14609568.2009.06699.x.

Grill-Spector, K., Kourtzi, Z. \& Kanwisher, N. (2001). The lateral occipital complex and its role in object recognition. Vision Res., 41(10-11), 1409-1422. doi: 10.1016/S00426989(01)00073-6

Hebart, M.N. \& Baker, C.I. (2017). Deconstructing multivariate decoding for the study of brain function. NeuroImage, 180, 4-18. doi: 10.1016/j.neuroimage.2017.08.005

Hupé, J.M. (2015). Statistical inferences under the Null hypothesis: Common mistakes and pitfalls in neuroimaging studies. Front. Neurosci., 9, 18. doi:

10.3389/fnins. 2015.00018

Hupé, J.M. (2017). Comment on "Ducklings imprint on the relational concept of 'same or different"”. Science, 355(6327), 806-806. doi: 10.1126/science.aah6047

Hupé, J.M., Bordier, C. \& Dojat, M. (2012a). A BOLD signature of eyeblinks in the visual cortex. NeuroImage, 61, 149-161. doi: 10.1016/j.neuroimage.2012.03.001

Hupé, J.M., Bordier, C. \& Dojat, M. (2012b). The neural bases of grapheme-color synesthesia are not localized in real color sensitive areas. Cereb. Cortex, 22, 1622-1633. doi: 10.1093/cercor/bhr236 
Hupé, J.M. \& Dojat, M. (2015). A critical review of the neuroimaging literature on synesthesia. Front. Hum. Neurosci., 9, 103. doi: 10.3389/fnhum.2015.00103

Janik McErlean, A.B. \& Banissy, M.J. (2017). Color processing in synesthesia: what synesthesia can and cannot tell us about mechanisms of color processing. Top. Cogn. Sci., 9(1), 215-227. doi: 10.1111/tops. 12237

Kriegeskorte, N., Goebel, R. \& Bandettini, P. (2006). Information-based functional brain mapping. Proc. Natl. Acad. Sci. USA, 103(10), 3863-3868. doi: 10.1073/pnas.0600244103

Kruschke, J.K. (2015). Doing Bayesian data analysis, Second Edition: A tutorial with R, JAGS, and Stan. Burlington, MA: Academic Press / Elsevier.

Kruschke, J.K. \& Liddell, T.M. (2018). The Bayesian New Statistics: Hypothesis testing, estimation, meta-analysis, and power analysis from a Bayesian perspective. Psychon. Bull. Rev., 25, 178-206. doi: 10.3758/s13423-016-1221-4

Lorenz, S., Weiner, K.S., Caspers, J., Mohlberg, H., Schleicher, A., Bludau, S., Eickhoff, S.B., Grill-Spector, K., Zilles, K. \& Amunts, K. (2017). Two new cytoarchitectonic areas on the human mid-fusiform gyrus. Cereb. Cortex, 27(1), 373-385. doi: 10.1093/cercor/bhv225

Misaki, M., Kim, Y., Bandettini, P.A. \& Kriegeskorte, N. (2010). Comparison of multivariate classifiers and response normalizations for pattern-information fMRI. Neuroimage, $\mathbf{5 3 ,}$ 103-118. doi: 10.1016/j.neuroimage.2010.05.051

Mumford, J.A., Turner, B.O., Ashby, F.G. \& Poldrack, R.A. (2012). Deconvolving BOLD activation in event-related designs for multivoxel pattern classification analyses. NeuroImage, 59(3), 2636-2643. doi: 10.1016/j.neuroimage.2011.08.076 
Norman, K.A., Polyn, S.M., Detre, G.J. \& Haxby, J.V. (2006). Beyond mind-reading: multivoxel pattern analysis of fMRI data. Trends Cogn. Sci., 10(9), 424-430. doi: 10.1016/j.tics.2006.07.005

Parkes, L.M., Marsman, J.B., Oxley, D.C., Goulermas, J.Y. \& Wuerger, S.M. (2009). Multivoxel fMRI analysis of color tuning in human primary visual cortex. J. Vis., 9(1), 1 1-13. doi: 10.1167/9.1.1

Pedregosa, F., Varoquaux, G., Gramfort, A., Michel, V., Thirion, B., Grisel, O., Blondel, M., Prettenhofer, P., Weiss, R., Dubourg, V., Vanderplas, J., Passos, A., Cournapeau, D., Brucher, M., Perrot, M. \& Duchesnay, E. (2011). Scikit-learn: Machine Learning in Python. J. Mach. Learn. Res., 12, 2825-2830.

Poldrack, R.A. (2007). Region of interest analysis for fMRI. Soc. Cogn. Affect. Neurosci., 2(1), 67-70. doi: 10.1093/scan/nsm006

Reddy, L., Tsuchiya, N. \& Serre, T. (2010). Reading the mind's eye: decoding category information during mental imagery. NeuroImage, 50(2), 818-825. doi: 10.1016/j.neuroimage.2009.11.084

Reeder, R.R. (2016). Individual differences shape the content of visual representations. Vision Res., 141, 266-281, doi: 10.1016/j.visres.2016.08.008

Rouw, R. \& Scholte, H.S. (2010). Neural basis of individual differences in synesthetic experiences. J. Neurosci., 30(18), 6205-6213. doi: 10.1523/JNEUROSCI.344409.2010

Rouw, R. \& Scholte, H.S. (2016). Personality and cognitive profiles of a general synesthetic trait. Neuropsychologia, 88, 35-48. doi: 10.1016/j.neuropsychologia.2016.01.006

Ruiz, M.J. (2014). Codage Cortical de la Synesthésie Graphème-Couleur (Doctoral dissertation, Université de Grenoble), https://hal-univ-tlse3.archives-ouvertes.fr/tel01091142 
Ruiz, M.J. \& Hupé, J.M. (2015). Assessment of the hemispheric lateralization of graphemecolor synesthesia with Stroop-type tests. PLoS ONE, 10(3), e0119377. doi: 10.1371/journal.pone.0119377

Ruiz, M.J., Hupé, J.M. \& Dojat, M. (2012). Use of pattern-information analysis in vision science: a pragmatic examination. In: Wang F., Shen D., Yan P., Suzuki K. (eds), Machine Learning in Medical Imaging. MLMI 2012. Lecture Notes in Computer Science, Vol. 7588. Springer, Berlin, Heidelberg, pp. 103-110. doi: 10.1007/978-3642-35428-1_13

Scheperjans, F., Hermann, K., Eickhoff, S.B., Amunts, K., Schleicher, A. \& Zilles, K. (2008). Observer-independent cytoarchitectonic mapping of the human superior parietal cortex. Cereb. Cortex, 18(4), 846-867. doi: 10.1093/cercor/bhm116

Shifferman, E. (2015), More than meets the fMRI: the unethical apotheosis of neuroimages. $J$. Cogn. Neuroethol, 3, 57-116.

Simner, J. \& Carmichael, D.A. (2015). Is synaesthesia a dominantly female trait? Cogn. Neurosci., 6(2-3), 68-76. doi: 10.1080/17588928.2015.1019441

Simner, J., Mulvenna, C., Sagiv, N., Tsakanikos, E., Witherby, S.A., Fraser, C., Scott, K. \& Ward, J. (2006). Synaesthesia: The prevalence of atypical cross-modal experiences. Perception, 35(8), 1024-1033. doi: 10.1068/p5469

Stelzer, J., Lohmann, G., Mueller, K., Buschmann, T. \& Turner, R. (2014). Deficient approaches to human neuroimaging. Front. Hum. Neurosci., 8(462). doi: 10.3389/fnhum.2014.00462

Stokes, M., Thompson, R., Cusack, R. \& Duncan, J. (2009) Top-down activation of shapespecific population codes in visual cortex during mental imagery. J. Neurosci., 29, 1565-1572. doi: 10.1523/JNEUROSCI.4657-08.2009 
Stoughton, C.M. \& Conway, B.R. (2008) Neural basis for unique hues. Curr. Biol., 18, R698699. doi: 10.1016/j.cub.2008.06.018

Thirion, B., Duchesnay, E., Hubbard, E., Dubois, J., Poline, J.B., Lebihan, D. \& Dehaene, S. (2006). Inverse retinotopy: inferring the visual content of images from brain activation patterns. NeuroImage, 33(4), 1104-1116. doi: 10.1016/j.neuroimage.2006.06.062

Tootell, R.B.H. \& Nasr, S. (2017). Columnar segregation of magnocellular and parvocellular streams in human extrastriate cortex. J. Neurosci., 37(33), 8014-8032. doi: 10.1523/jneurosci.0690-17.2017

Turner, R. (2016). Uses, misuses, new uses and fundamental limitations of magnetic resonance imaging in cognitive science. Philos. Trans. R. Soc. Lond. B. Biol. Sci., 371(1705). doi: 10.1098/rstb.2015.0349

Vasseur, F., Delon-Martin, C., Bordier, C., Warnking, J., Lamalle, L., Segebarth, C. \& Dojat, M. (2010). fMRI retinotopic mapping at 3 T: Benefits gained from correcting the spatial distortions due to static field inhomogeneity. J. Vis., $\mathbf{1 0}$ (12), 30. doi: $10.1167 / 10.12 .30$

Ward, J. (2013). Synesthesia. Annual Reviews in Psychology, 64, 49-75. doi: 10.1146/annurev-psych-113011-143840

Wasserstein, R.L. \& Lazar, N.A. (2016). The ASA's Statement on p-Values: Context, Process, and Purpose. The American Statistician, 70(2), 129-133. doi: $10.1080 / 00031305.2016 .1154108$

Watson, M.R., Chromy, J., Crawford, L., Eagleman, D.M., Enns, J.T. \& Akins, K.A. (2017). The prevalence of synaesthesia depends on early language learning. Conscious Cogn., 48, 212-231. doi: 10.1016/j.concog.2016.12.004

Witthoft, N. \& Winawer, J. (2013). Learning, memory, and synesthesia. Psychol. Sci., 24(3), 258-265. doi: 10.1177/0956797612452573 
Yarkoni, T., Poldrack, R.A., Van Essen, D.C. \& Wager, T.D. (2010). Cognitive neuroscience 2.0: building a cumulative science of human brain function. Trends Cogn. Sci., 14(11), 489-496. doi: 10.1016/j.tics.2010.08.004 


\section{Appendix}

Supporting Information is available. It contains:

Table S1. Clusters identified based on whole brain analyses and tested post-hoc with MVPA.

Figure S1. Right occipito-parietal cortex cluster identified based on whole brain univariate analysis

Figure S2. Left anterior insula cluster identified based on whole brain univariate analysis

Figure S3. Right frontal cortex cluster identified based on whole brain univariate analysis

Figure S4. Alternative version of Figure 6, based on mixed-effect generalized linear models

Figure S5. Alternative version of Figure 7, based on mixed-effect generalized linear models 
Table S1. Clusters identified based on whole brain analyses and tested post-hoc with MVPA

\begin{tabular}{|c|c|c|c|c|c|c|c|c|c|c|c|c|c|c|c|c|c|c|}
\hline \multicolumn{5}{|c|}{ Whole brain method to identify clusters } & & & & \multicolumn{5}{|c|}{ MVPA tests in post-hoc clusters } & \multirow[b]{2}{*}{ C2S } & & \multirow[b]{2}{*}{ S2C } & & \multirow[b]{2}{*}{ g1g2 } & \multirow{3}{*}{$s>0,25$} \\
\hline & & & & & & & & & Col & & Syn & & & & & & & \\
\hline analysis & stimuli & stat & comparison & contrast & size & MNI XYZ & name & & $S>C$ & $S>0.25$ & $S>C$ & $S>0.25$ & $S>C$ & $S>0.25$ & $S>C$ & $S>0.25$ & $S>C$ & \\
\hline \multirow[t]{3}{*}{ univariate } & graph. & $\mathrm{T}$ & P-s & Syn $>$ Con & 314 & {$\left[\begin{array}{llll}-60 & -4 & 21\end{array}\right]$} & left precentral & yes & & & & & & & & & & \\
\hline & & $\mathrm{T}$ & P-s \& 2-s & Con>Syn & & & & & & & & & & & & & & \\
\hline & & $\mathrm{F}$ & P-s \& 2-s & Syn $>$ Con & & & & & & & & & & & & & & \\
\hline \multirow[t]{3}{*}{ Fig. S1 } & & $\mathrm{F}$ & P-s \& 2-s & Con>Syn & 388 & {$\left[\begin{array}{llll}33 & -70 & 33\end{array}\right]$} & right occipital-parietal & yes & 0.011 & 0.003 & 0.016 & 0.122 & & & & & & \\
\hline & & & P-S & Con>Syn & 361 & {$\left[\begin{array}{llll}-24 & 21 & -8\end{array}\right]$} & left insula & yes & & & & & & & & & & \\
\hline & colours & $T$ & P-s & Syn $>$ Con & 327 & {$[-39-16-8]$} & left posterior insula & yes & & & & & & & & & & \\
\hline \multirow[t]{3}{*}{ Fig. S2 } & & & P-s & Syn $>$ Con & 203 & {$\left[\begin{array}{lll}-35 & 35 & -3\end{array}\right]$} & left anterior insula & yes & & & & & & & 0.029 & 0.077 & & \\
\hline & & & P-S & Syn $>$ Con & 142 & {$\left[\begin{array}{llll}-32 & -16 & -33\end{array}\right]$} & left parahippocampal & yes & & & & & & & & & & \\
\hline & & & $2-s$ & Syn $>$ Con & 513 & {$[47-616]$} & right middle temporal & yes & & & & & & & & & & \\
\hline \multirow[t]{4}{*}{ Fig. S3 } & & & 2-s & Syn $>$ Con & 385 & {$\left[\begin{array}{llll}5 & 30 & 42\end{array}\right]$} & right superior, frontal & yes & & & 0.023 & 0.087 & & & & & & \\
\hline & & $\mathrm{T}$ & P-S \& 2-5 & Con>Syn & & & & & & & & & & & & & & \\
\hline & & $\mathrm{F}$ & P-s \& 2-s & Syn $>$ Con & & & & & & & & & & & & & & \\
\hline & & $F$ & P-s & Con>Syn & 128 & {$\left[\begin{array}{lll}-17 & 18 & 21\end{array}\right]$} & white matter & no & & & & & & & & & & \\
\hline \multirow[t]{15}{*}{ MVPA } & colours & Col & P-s \& 2-s & Syn $>$ Con & & & & & & & & & & & & & & \\
\hline & & Col & P-S \& 2-S & Con>Syn & & & & & & & & & & & & & & \\
\hline & graph. & Syn & P-s & Syn $>$ Con & 459 & {$\left[\begin{array}{lll}24 & -43 & 44\end{array}\right]$} & right parietal & yes & & & $4.10^{-7}$ & & & & & & & \\
\hline & & & 2-s & Syn $>$ Con & 351 & {$\left[\begin{array}{llll}-24 & -40 & 53\end{array}\right]$} & left parietal & yes & & & $2.10^{-4}$ & & & & & & & \\
\hline & & Syn & 1-s & Syn $>0.25$ & & & & & & & & & & & & & & \\
\hline & & Syn & P-S \& 2-S & Con>Syn & & & & & & & & & & & & & & \\
\hline & & Syn & $1-s$ & Con $>0.25$ & & & & & & & & & & & & & & \\
\hline & all & $\mathrm{C} 2 \mathrm{~S}$ & P-s \& 2-S & Syn>Con & & & & & & & & & & & & & & \\
\hline & & $\mathrm{C} 2 \mathrm{~S}$ & $1-s$ & Syn $>0.25$ & & & & & & & & & & & & & & \\
\hline & & $\mathrm{C} 2 \mathrm{~S}$ & P-S \& 2-5 & Con>Syn & & & & & & & & & & & & & & \\
\hline & & $\mathrm{C} 2 \mathrm{~S}$ & $1-s$ & Con $>0.25$ & & & & & & & & & & & & & & \\
\hline & all & S2C & P-s & Syn $>$ Con & 297 & {$\left[\begin{array}{lll}39 & -70 & 2\end{array}\right]$} & right occipito-temporal & yes & & & & & & & $7.10^{-7}$ & $1.10^{-5}$ & & \\
\hline & & & 2-s & Syn $>$ Con & 459 & [39-73 5] & right occipito-temporal & yes & & & & & & & $6.10^{-6}$ & $7.10^{-6}$ & & \\
\hline & & & 2-s & Syn $>$ Con & 648 & {$\left[\begin{array}{lll}-27 & -1 & -7\end{array}\right]$} & left putamen & yes & & & & & & & $3.10^{-4}$ & $7.10^{-4}$ & & \\
\hline & & $\mathrm{S} 2 \mathrm{C}$ & 1-s & Syn $>0.25$ & 486 & {$\left[\begin{array}{lll}42 & -73 & 2\end{array}\right]$} & right occipito-temporal & yes & & & & & & & $9.10^{-6}$ & $5.10^{-6}$ & & \\
\hline \multirow[t]{7}{*}{ Fig. 9} & & & 1-s & Syn $>0.25$ & 729 & {$\left[\begin{array}{llll}-33 & -28 & 50]\end{array}\right.$} & left parietal & yes & & & 0.012 & 0.004 & & & \multirow[t]{2}{*}{$5.10^{-4}$} & $2.10^{-6}$ & & \\
\hline & & S2C & P-s \& 2-s & Con>Syn & & & & & & & & & & & & & & \\
\hline & & $\mathrm{S} 2 \mathrm{C}$ & $1-s$ & Con $>0.25$ & & & & & & & & & & & & & & \\
\hline & graph. & g1g2 & P-s \& 2-s & Syn>Con & & & & & & & & & & & & & & \\
\hline & & g1g2 & $1-s$ & Syn $>0.25$ & & & & & & & & & & & & & & \\
\hline & & g1g2 & P-s \& 2-5 & Con>Syn & 837 & {$\left[\begin{array}{llll}-42 & 20 & 26\end{array}\right]$} & left inferior frontal & no & & & & & & & & & & \\
\hline & & g1g2 & $1-s$ & Con $>0.25$ & & & & & & & & & & & & & & \\
\hline
\end{tabular}

Clusters potentially involved in synaesthesia were identified based on whole brain univariate analysis and searchlight MVPA. For each analysis the line in the table indicates which stimuli were presented ('graph.': achromatic letters and digits; 'all': MVPA based on both graphemes and coloured rings), which statistics (stat) was used to create individual whole brain maps (first-level analysis), the statistical test (comparison: $\mathrm{P}$-s $=$ paired-sample $T$-test; 2 -s $=$ two-sample $T$-test; 1 -s $=$ one-sample $T$-test) performed for the second level analysis as well as the statistical contrast. For all individual statistical maps (first level analysis), we applied a spatial smoothing with FWHM $=9 \mathrm{~mm}$ for univariate analyses and no smoothing for MVPA. For second-level analyses, the cluster forming threshold was set at $P=$ 0.001 . We list all clusters significant at $P_{F W E}<0.05$, their size in $\mathrm{mm}^{3}$ (voxel size was $1.5 \mathrm{~mm}^{3}$ for univariate analyses and $3 \mathrm{~mm}^{3}$ for multivariate analyses), the coordinates in the $M N I$ space of the voxel with the smallest $P$-value in the cluster as well as the name used in the main text, corresponding to their approximate location. Empty lines mean the absence of any significant cluster. Grey font was used for statistical contrasts for which we did not have any reason to expect any difference. The right part of the table lists the comparisons of MVPA scores within these post-hoc clusters. The names of the MVPA classifiers are explained in Figure 3. We compared the scores of synaesthetes and controls with paired $T$-tests and report the $P$-values that were below 0.05 (two-sided tests, not corrected for multiple comparisons; the results of two-sample $T$-tests were similar). The scores of controls were never significantly larger than the scores of synaesthetes. We also tested the scores of synaesthetes against chance (two-sided one-sample tests) and reported $P$-values systematically when there was a difference 
between synaesthetes and controls. For the results of MVPA tests in clusters defined by the whole brain MVPA searchlight, we shaded in grey the cells corresponding to circular analysis. Note that for the results of the 'S2C' classifier in clusters based on 'S2C', the comparison of synaesthetes and controls and the comparison of synaesthetes against chance are not independent (the scores of controls in these cluster were in fact on average below chance). 


\section{Figure S1. Right occipito-parietal cortex cluster identified based on whole brain univariate analysis}
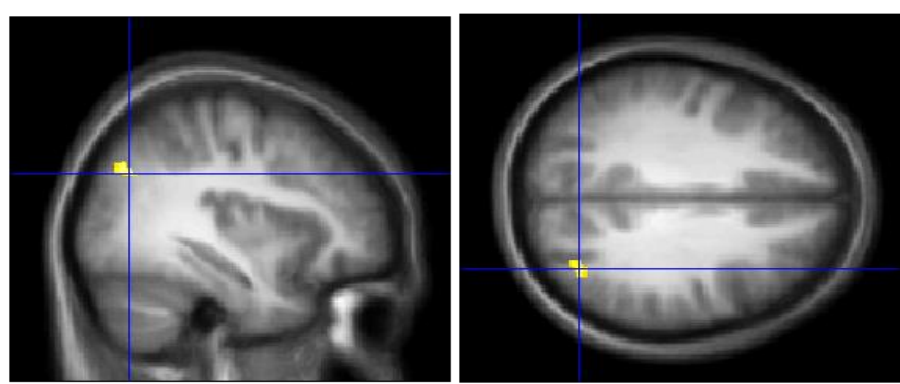

The univariate analysis of the $F$-contrast for achromatic graphemes revealed a significant cluster $\left(P_{F W E}\right.$ $<0.05)$ in the right occipito-parietal cortex (MNI XYZ = [33 -70 33], $\mathrm{k}=111)$ for the contrast Con>Syn (paired $T$-test). MVPA tests in this cluster revealed a higher score in synaesthetes than controls for the 'Syn' classifier, decoding graphemes based on training on graphemes (95\% CI of the difference $[1.4,11.3] \%)$. The decoding performance for synaesthetes was also slightly above chance $(95 \%$ CI $[24,31] \%)$ but did not correlate with photism strength $(P=0.67)$. (This result is paradoxical since the modulation by graphemes was higher in controls - that's how the ROI was defined - so differences of BOLD signals could have favoured the 'Syn' classifier for controls). In this cluster, scores were also higher in synaesthetes than controls for the 'Col' classifier, decoding colours based on training on colours $(95 \% \mathrm{CI}$ of the difference $[0.9,6.1] \%)$. The decoding performance for synaesthetes was also significantly above chance $(95 \%$ CI $[26,30] \%)$ but did not correlate with photism strength $(P=0.66)$. 
Figure S2. Left anterior insula cluster identified based on whole brain univariate analysis
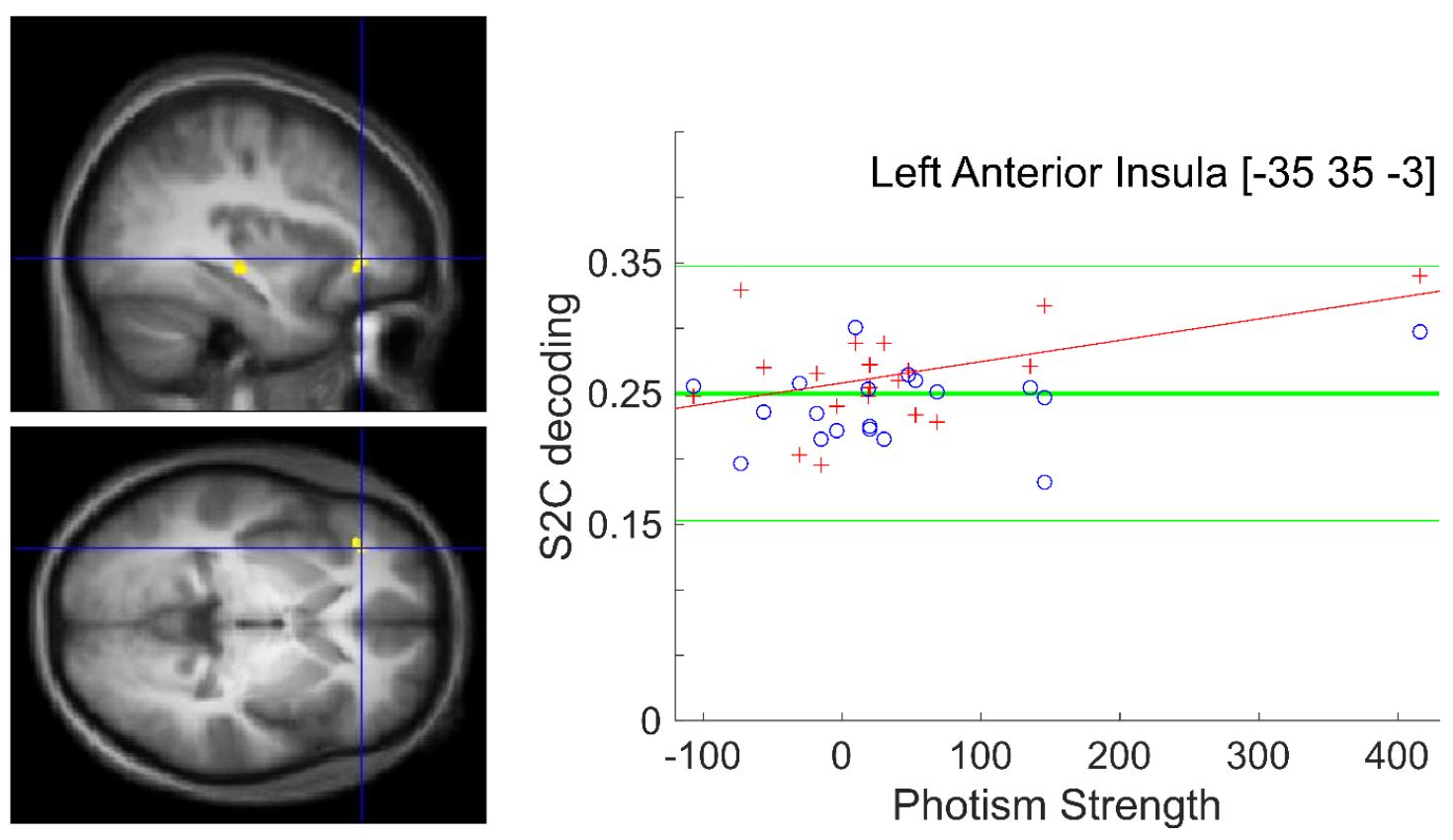

The univariate analysis of the $T$-contrast for colour rings revealed a significant cluster $\left(P_{F W E}<0.05\right)$ in the left anterior insula (MNI XYZ $=\left[\begin{array}{lll}-35 & 35 & -3\end{array}\right], \mathrm{k}=60$ ) for the contrast $\mathrm{Syn}>\mathrm{Con}$ (paired $T$-test). MVPA tests in this cluster revealed that the ' $\mathrm{S} 2 \mathrm{C}$ ' classifier of synaesthetes decoded colours better than controls based on training on graphemes (95\% CI of the difference [0.3, 4.6]\%). The decoding performance for synaesthetes was also slightly above chance (95\% CI [25, 28]\%) and slightly correlated with the strength of synaesthetic associations (same conventions as in Figure 8). However, the correlation is driven by only one data point (non-parametric Spearman test on ranks, $P=0.30$ ). 
Figure S3. Right frontal cortex cluster identified based on whole brain univariate analysis
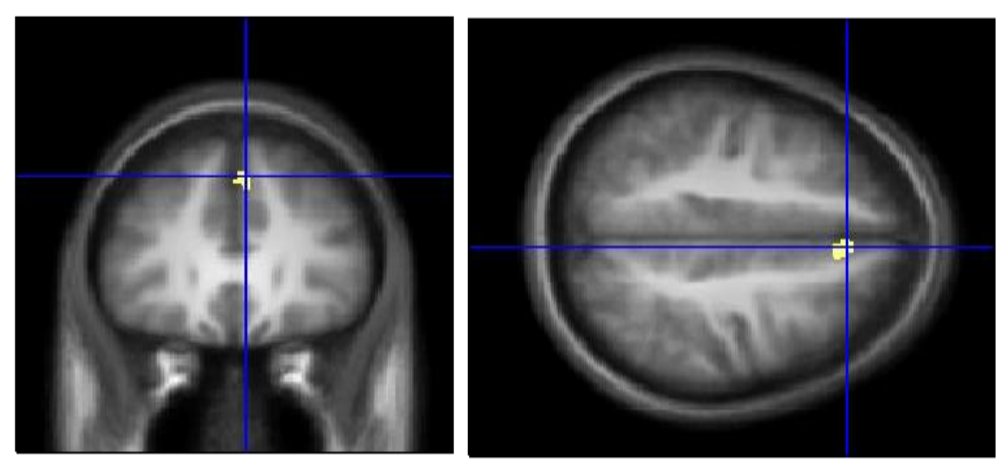

The univariate analysis of $T$-contrast for colour rings revealed a significant cluster $\left(P_{F W E}<0.05\right)$ in the right frontal cortex (MNI XYZ $=\left[\begin{array}{ll}5 & 30\end{array} 42\right], \mathrm{k}=114$ ) for the contrast $\mathrm{Syn}>\mathrm{Con}$ (two-sample $T$-test). MVPA tests in this cluster revealed that the 'Syn' classifier of synaesthetes decoded graphemes better than controls based on training on graphemes $(95 \% \mathrm{CI}$ of the difference $[1,12] \%)$. The decoding performance for synaesthetes was also slightly above chance (95\% CI [24, 34]\%) but did not correlate with photism strength $(P=0.54)$. 

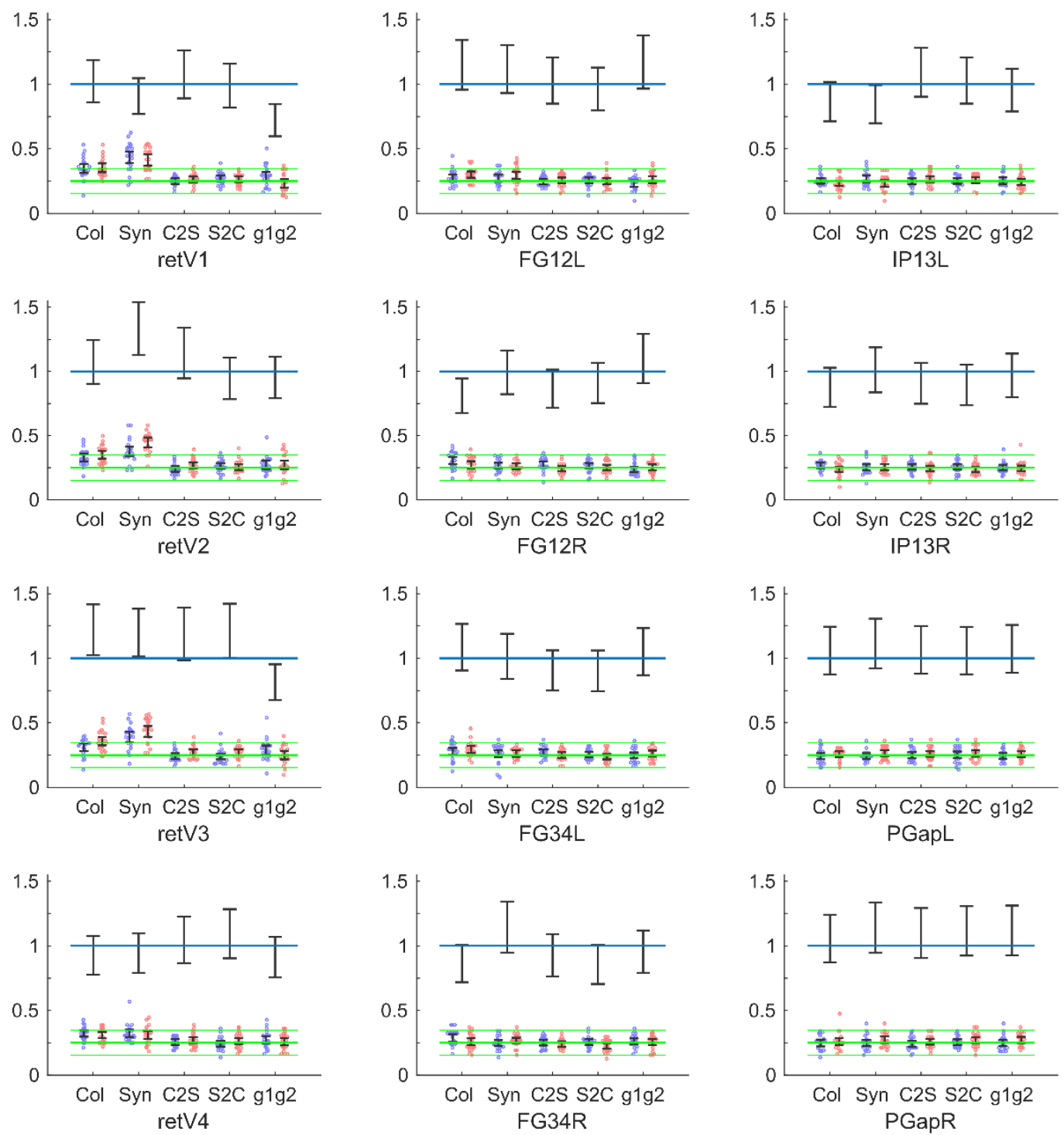

Here the difference of performance between synaesthetes and controls was estimated by a mixedeffect generalized linear models with a binomial family and a logit link function. The y-axis represents therefore not only the performance of classifiers for individual subjects and their group average and CI like in Figure 6, but also the odd-ratio of synaesthetes against their matched controls $(1=$ no difference between groups, blue line; whiskers denote $95 \% \mathrm{CI}$ ). Estimation is slightly more precise with this more powerful analysis. 
Figure S5. Alternative version of Figure 7, based on mixed-effect generalized linear models
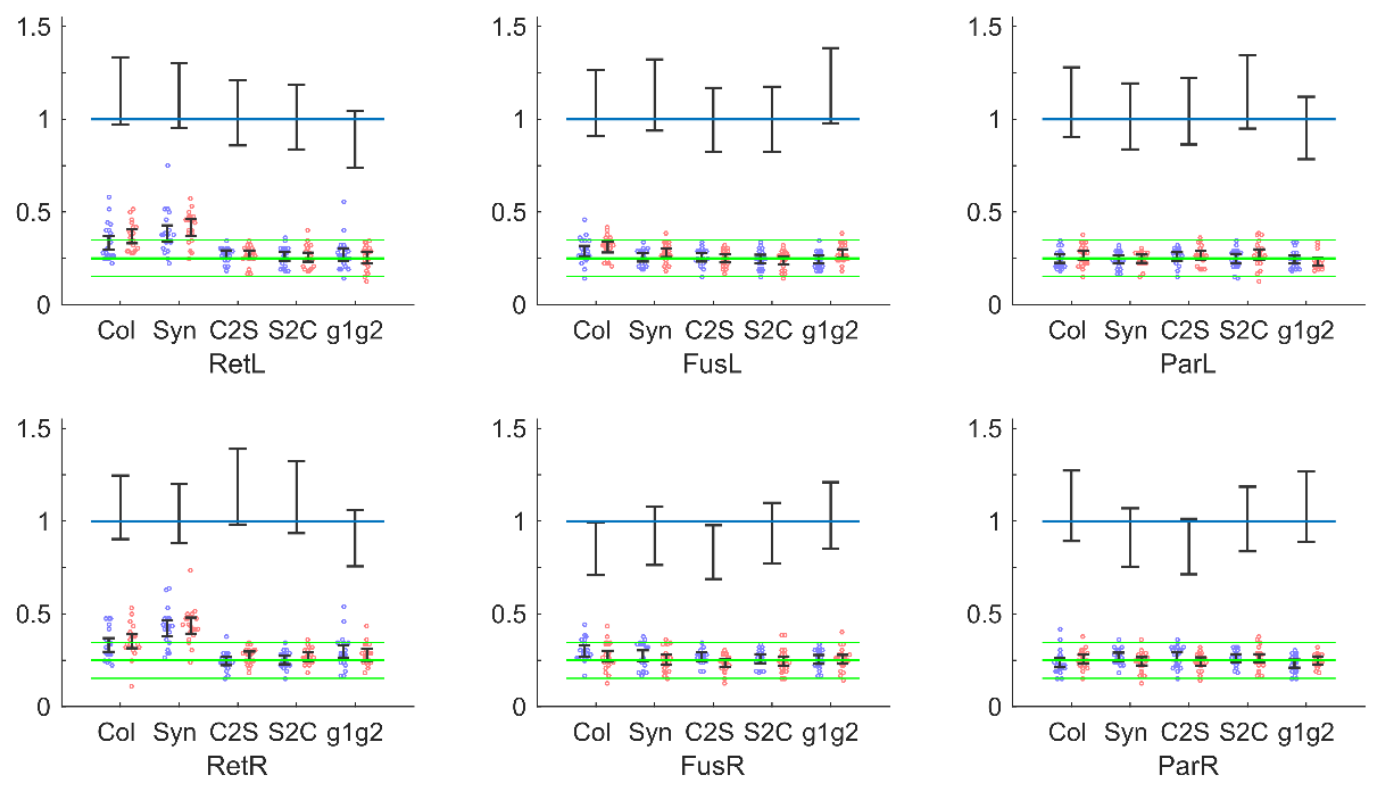